\title{
1 Bacterial retrons encode tripartite toxin/antitoxin systems
}

2

Jacob Bobonis ${ }^{1,2}$, André Mateus ${ }^{1}$, Birgit Pfalz ${ }^{1}$, Sarela Garcia-Santamarina ${ }^{1}$, Marco Galardini ${ }^{3}$, Callie Kobayashi ${ }^{4}$, Frank Stein ${ }^{5}$, Mikhail M. Savitski ${ }^{1,5,6}$, Johanna R. Elfenbein ${ }^{7,8^{*}}$, Helene Andrews-Polymenis ${ }^{4^{*}}$ and Athanasios Typas ${ }^{1,6^{*}}$

*Correspondence: jelfenbein@wisc.edu, handrews@tamu.edu \& typas@embl.de

1 European Molecular Biology Laboratory, Genome Biology Unit, Heidelberg, Germany ${ }^{2}$ Collaboration for joint PhD degree between EMBL and Heidelberg University, Faculty of Biosciences 3 European Molecular Biology Laboratory, European Bioinformatics Institute, Hinxton, UK ${ }^{4}$ Texas A\&M University, Department of Microbial Pathogenesis and Immunology, Texas, USA ${ }^{5}$ European Molecular Biology Laboratory, Proteomics Core Facility, Heidelberg, Germany ${ }^{6}$ European Molecular Biology Laboratory, Structural and Computational Biology Unit, Heidelberg, Germany

${ }^{7}$ University of Wisconsin-Madison, Department of Pathobiological Sciences, Madison, WI, USA

${ }^{8}$ North Carolina State University, Department of Clinical Sciences, Raleigh, NC, USA

\section{ABSTRACT}

Retrons are genetic retroelements, commonly found in bacterial genomes and recently repurposed as genome editing tools. Their encoded reverse transcriptase (RT) produces a multi-copy single-stranded DNA (msDNA). Despite our understanding of their complex biosynthesis, the function of msDNAs and therefore, the physiological role of retrons has remained elusive. We establish that the retron-Sen2 in Salmonella Typhimurium encodes a toxin, which we have renamed as RcaT (Retron cold-anaerobic Toxin). RcaT is activated when msDNA biosynthesis is perturbed and its toxicity is higher at ambient temperatures or during anaerobiosis. The RT and msDNA form together the antitoxin unit, with the RT binding RcaT, and the msDNA enabling the antitoxin activity. Using another $E$. coli retron, we establish that this toxin/antitoxin function is conserved, and that RT-toxin interactions are cognate. Altogether, retrons constitute a novel family of tripartite toxin/antitoxin systems. 


\section{INTRODUCTION}

Reverse transcriptases (RTs), thought initially to be unique in retroviruses, were first discovered three decades ago in bacteria, encoded in genetic elements named retrons ${ }^{1,2}$. Retrons were originally identified because they produce multiple copies of satellite DNA molecules, called msDNA (multi-copy single-stranded DNA) ${ }^{3}$. They are present in bacteria across the phylogenetic tree ${ }^{4}$. The ability of retrons to produce high-quantities of singlestranded DNA in situ has been recently exploited for recombineering and other genome-editing approaches ${ }^{5-8}$. Yet, the natural function of retrons has remained enigmatic.

In contrast to their function, the msDNA biogenesis pathway is well understood. Retrons contain a non-coding RNA gene ( $m s r m s d$ ), the RT, and often, accessory genes of unknown function ${ }^{9}$ (Fig. 1A - here the retron-Sen2 [retron-ST85] depicted as example). RTs reverse transcribe their cognate msrmsd-RNA, utilising the msr-RNA as primer, and the msd-RNA as template ${ }^{10}$ (Fig. 1B). While the msd-DNA is being reverse-transcribed, the msd-RNA template is degraded by ribonuclease $\mathrm{H}($ RNAse $H){ }^{10}$ (Fig. 1A-B). The end product is usually a branched DNA/RNA hybrid, with the msd-DNA being covalently joined to the msr-RNA through a 2'-5' phosphodiester bond made by the RT ${ }^{11}$ (Fig. 1B). Some msDNAs (e.g., msDNA-Sen2) are pure unbranched DNA ${ }^{12,13}$, with the DNA branch being separated from the RNA-branch by the housekeeping Exonuclease VII ${ }^{14}$ (Exo VII; encoded by $x \operatorname{se} A$ and $x \operatorname{se} B$ genes; Fig. $1 \mathrm{~A}$ ). Exo VII cleaves four deoxyribonucleotides from the msDNA, separating it from its branched precursor (Fig. 1B). Both branched ${ }^{15}$ and unbranched ${ }^{16}$ msDNAs can remain in complex with their cognate RTs (Fig. 1B). The accessory proteins (e.g., STM14_4640 in retron-Sen2 ${ }^{17}$, Fig. $1 \mathrm{~A}$ ), do not affect msDNA biosynthesis ${ }^{13,18}$, and neither their sequence nor the position in retron elements are conserved.

The bottleneck to understand the natural function of retrons has been the absence of phenotypes associated with retron deletions. The first retron-deletion phenotype was reported for retron-Sen2 of Salmonella enterica subsp. enterica ser. Typhimurium str. 14028s (STm), wherein the RT-Sen2 was found to be essential for STm survival in calves ${ }^{18}$. This was because it allows STm to grow in anaerobic conditions, present in calf intestines ${ }^{19}$. Here, we report that retron-Sen2 deletion mutants are also unable to grow at lower temperatures. By exploiting the retron cold-sensitivity phenotype, we show that the retron-Sen2 accessory gene STM14_4640 ( $r c a T)$ encodes a bona fide toxin. Perturbing msDNA biosynthesis at any stage results in toxin activation, and thereby, growth inhibition in anaerobic conditions and cold. Although reminiscent of Toxin/Antitoxin (TA) systems, which are composed of a protein or RNA antitoxin

and cognate toxin ${ }^{20}$, retron-Sen2 forms a novel tripartite TA system: RcaT is the toxin, and the RT-msDNA complex is the antitoxin. Using another retron encoded by E. coli NILS-16 ${ }^{21}$, 
retron-Eco9, we demonstrate that this TA function is conserved and that the RT provides specificity to the TA system. We propose that bacterial retrons function as TA systems, where the RT-msDNA antitoxins directly inhibit retron-encoded toxins, by forming inactive msDNART-toxin complexes.

\section{RESULTS}

\section{Perturbations in msDNA-Sen2 biosynthesis inhibit STm growth in cold}

As part of a larger chemical-genetics effort, we profiled the fitness of a single-gene deletion STm library ${ }^{22}$ across hundreds of conditions (unpublished data). The two gene deletions that led to the highest growth sensitivity at room temperature (cold-sensitivity) were $\Delta r r t T$ ( $\triangle R T$ Sen2) and $\Delta x \operatorname{se} A$ (Fig. $1 \mathrm{C}$ ). Both RT-Sen2 and Exo VII are involved in msDNA-Sen2 biogenesis ${ }^{14,18}$ (Fig. 1A-1B). To validate these results and exclude confounding effects (secondary mutations or polar effects), we transferred these deletions, as well as that of the accessory gene STM14_4640 (Fig. 1A), to a clean STm genetic background and flipped out the antibiotic resistance cassettes. We also constructed new marker-less deletions for the remaining msDNA-Sen2 biosynthesis genes that were not part of the library $(r n h A, x s e B$, and msrmsd; Fig. 1A-B). All gene deletions, except for $\triangle$ STM14_4640, led to severely restricted growth at $15^{\circ} \mathrm{C}$ (Fig. 1D) and temperatures up to $25^{\circ} \mathrm{C}$ (ED Fig. 1A). Consistent with previous data on $\Delta r r t T$ and $\Delta m s d^{18}$, all mutants, except for $\Delta S T M 14 \_4640$, also grew slower under anaerobic conditions at $37^{\circ} \mathrm{C}$ (ED Fig. 1B-C). It is known that msDNA-Sen2 cannot be produced in $\Delta r r t T$ and $\Delta m s r m s d$ strains ${ }^{18}$. We observed that RNAse $\mathrm{H}(r n h A)$ and Exo VII (xseA/xseB), but not STM14_4640, were also required for biosynthesis of mature msDNASen2 (ED Fig. 1D). In summary, any perturbation in the msDNA-Sen2 biosynthesis pathway impairs STm growth at lower temperatures and anaerobiosis.

\section{Retron-Sen2 encodes a toxin, which is activated upon msDNA biogenesis perturbations}

To understand why retron-Sen2 mutants are cold-sensitive, we isolated and sequenced spontaneous suppressors of $\Delta r r t T, \Delta x s e A$, and $\Delta m s r m s d$ mutants, that restored growth at $15^{\circ} \mathrm{C}$. 28 out of 29 suppressor mutations mapped in STM14_4640, the retron-encoded accessory gene (Fig. 2A). Mutations included frameshifts (14/29), premature termination sites (4/29), or single amino acid substitutions (10/29) in STM14_4640 (ED Fig. 2A). One residue, D296, was mutated to different amino acids in all three mutant backgrounds, suggesting that it is key for the function of STM14_4640. The only suppressor that did not map in STM14_4640, had a 62 base-pair deletion within the msd region ( $\Delta 62: m s d)$ (ED Fig. 2A). To identify the minimal msd deletion-region that fully suppressed cold-sensitivity, we made progressive scarless deletions in msd (ED Fig. 2B). Deleting up to the 63 base-pair in msd ( $\Delta 63: m s d)$ was able to revert the cold-sensitivity phenotype (ED Fig. $2 \mathrm{C}$ ). However, all 
constructs that (partially) alleviated the cold-sensitivity, resulted also in down-regulation of the expression of STM14_4640 (ED Fig. 2D), which lies directly downstream. Hence coldsensitivity was linked in all cases to STM14_4640 integrity/expression. Therefore, we reasoned that in the absence of msDNA-Sen2, the product of STM14_4640 impairs growth at cold and anaerobic conditions. Indeed, deleting STM14_4640 in $\Delta x s e A, \Delta x s e B$, or $\Delta r n h A$ mutants, restored growth at cold temperatures (Fig. 2B) and in anaerobic conditions (Fig. 2C). We thus renamed STM14_4640 as rcaT (retron cold-anaerobic Ioxin).

In the absence of msDNA, RcaT inhibited growth at $15^{\circ} \mathrm{C}$ (ED Fig. $3 \mathrm{~A}$ ), and its effect was bacteriostatic (ED Fig. 3B). Consistent with RcaT acting as a bona fide toxin, ectopically expressing RcaT, but not its inactive RcaT-D296V form, was toxic in Escherichia coli even at $37^{\circ} \mathrm{C}$ (Fig. 2D). This toxicity was exacerbated at lower temperatures (ED Fig. 3C), likely reflecting an inherent property of RcaT to be more active in cold. In these conditions, overexpressing RcaT was bactericidal (ED Fig. 3D).

Thus, growth effects that stem from impairing msDNA biosynthesis can be rescued by inactivating or down-regulating the retron-Sen2 accessory gene ( $r c a T)$, which encodes a toxin. This configuration is reminiscent of TA systems, where in the absence of the antitoxin, the adjacently encoded toxin inhibits bacterial growth. Consistently, toxins of TA systems are bacteriostatic at natural expression levels, but lead to killing when over-expressed ${ }^{23}$.

\section{Retron-Sen2 is a tripartite TA system with RT and msDNA forming the antitoxin unit}

Consistent with the retron-Sen2 being a novel TA system that encodes both the toxin and antitoxin activity, overexpressing the entire STm retron-Sen2 in E. coli (msrmsd-rcaT-rrtT; pretron) did not impact growth (ED Fig. 4A), as opposed to overexpressing RcaT alone, which was toxic (Fig. 2D). RcaT toxicity was also blocked by co-expressing msrmsd-rrtT in trans from a separate plasmid (ED Fig. 4B), excluding that the antitoxin activity is dependent on in cis regulation. Thus, the msrmsd and the RT-Sen2 are sufficient to counteract the RcaT toxicity, and the entire retron constitutes a TA system.

To delineate which retron component acts as the antitoxin (branched/mature msDNA, RT, msrmsd-RNA), we constructed plasmids with retrons that lacked a single component ( $p$-retron$\Delta r r t T, \Delta m s r m s d, m s r m s d^{m u t}$, and $\left.\Delta r c a T\right)$. The $m s r m s d^{\text {mut }}$ carries a single point mutation in the msr region, that abrogates the production of msDNA, but does not affect msrmsd-RNA expression (branching $\mathrm{G}$ mutation) ${ }^{11}$. Additionally, we used RNAse $\mathrm{H}(\Delta r n h A)$ and Exo VII $(\Delta x \operatorname{se} A / \Delta x \operatorname{se} B) E$. coli mutants to stop the msDNA biosynthesis at different steps. In all cases, deleting any retron component (except for $\Delta r c a T$ ) or any msDNA-biosynthesis-related gene, 
143

144

145

146

147

148

149

150

151

152

153

154

155

156

157

158

159

160

161

162

163

164

165

166

167

168

169

170

171

172

173

174

175

176

177

178

179

led to the same toxicity as overexpressing rcaT alone (ED Fig. 4A \& C). Thus, the antitoxin activity against RcaT requires the presence of all retron-components and/or the mature msDNA-Sen2.

To test whether the retron-antitoxin is counteracting RcaT by down-regulating its expression, we Flag-tagged rcaT in STm WT and antitoxin deletion strains, and quantified RcaT levels at $37^{\circ} \mathrm{C}$ and $20^{\circ} \mathrm{C}$. RcaT levels remained similar in all mutants, and if anything, only slightly decreased in the $\Delta r r t T$ background (ED Fig. $5 \mathrm{~A}-\mathrm{B}$ ). This is presumably due to mild polar effects of removing $r t T$, which is located directly downstream of rcaT. In all cases, RcaT-3xFlag was fully functional as toxin (ED Fig. 5C). Therefore, RcaT expression is not inhibited by the retronantitoxin, which would have led to higher RcaT levels in the mutants.

Having excluded that RcaT is counteracted by the retron-antitoxin at an expression level, we tested if this occurs by direct protein-protein interactions, as in type II TA systems. To do so, we affinity purified chromosomally encoded RT-3xFlag or RcaT-3xFlag, in wildtype and retron antitoxin-deletion STm strains ( $\Delta x s e A$, and $\Delta m s r m s d / \Delta m s d$ ), at $37^{\circ} \mathrm{C}$ and $20^{\circ} \mathrm{C}$, and quantified interacting proteins by quantitative mass-spectrometry (AP-qMS). Indeed, RT and RcaT strongly and reciprocally pulled down each other (Fig. 3 \& ED Fig. 6). Notably, the RT-RcaT interaction occurred independently of the presence/maturation of msDNA, or of the temperature the toxin is active in. Tagged RT-3xFlag was fully functional in inhibiting RcaT (ED Fig. 7), and did not alter RT or RcaT protein levels in the input samples, compared to WT (ED Fig. 8A). In contrast, tagging RcaT led to lower RT levels in the input samples (ED Fig. 8B), but RcaT-3xFlag remained functional as toxin (ED Fig. 5C). This likely explains the lower levels of RT enrichment in the RcaT-3xFlag pull-downs (Fig. 3C). Thus, RcaT and RT stably interact with each other independently of the presence or maturation of msDNA, and of temperature.

Although RcaT and RT interact in the absence of msDNA, mature msDNA production is essential for antitoxin activity. We thus wondered whether msDNA-protein interactions are involved in the antitoxin activity. Retron-RTs from different species have been previously shown to co-purify with their mature msDNA products ${ }^{15,16}$. In order to assess whether RTSen2 also interacts with msDNA-Sen2, we first purified an RT-Sen2-6xHis protein fusion upon concomitant $m s r m s d-S e n 2$ expression in E. coli (ED Fig. 9A). The RT-Sen2-6xHis version was functional, as it could counteract the RcaT toxicity (ED Fig. 9B). At a second stage, we isolated total DNA from the purified RT-Sen2-6xHis protein sample, which yielded both mature and unprocessed msDNA-Sen2 (ED Fig. 9C). Therefore, the RT-Sen2 and msDNA-Sen2 interact with each other and are required together for the antitoxin activity. 
180

181

182

183

184

185

186

187

188

189

190

191

192

193

194

195

196

197

198

199

200

201

202

203

204

205

206

207

208

209

210

211

212

213

214

215

216

The RT confers specificity to the antitoxin \& the msDNA modulates the antitoxin activity Antitoxins of TA systems are specific against their cognate toxins. Since the retron-antitoxin is composed by both the RT and msDNA, we wondered which part provides the antitoxin specificity. To address this, we reasoned we could use a different retron-TA, with evolutionary diverged retron-components, and swap the individual components between retrons to make retron chimeras. For this, we used a novel retron from E. coli NILS-16, a clinical E. coli isolate 21,24 , that we named retron-Eco9. Retron-Eco9 has an RT and an accessory gene, which are $49 \%$ and $43 \%$ identical to RT-Sen2 and RcaT-Sen2 at the protein level, respectively. msrEco9 and $m s d-E \operatorname{co} 9$ are $85 \%$ and $58 \%$ identical to their Sen2 counterparts at the nucleotide level (ED Fig. 10A), and the msDNA-Eco9 retains a similar overall structure to msDNA-Sen2 (ED Fig. 10B). As for retron-Sen2, expressing rcaT-Eco9 inhibited the growth of E. coli, while expressing the entire retron-Eco9 did not (ED Fig. 10C). In addition, the msDNA-Eco9 required RNase $\mathrm{H} /$ ExoVII for its production and function (ED Fig. 10D-E). Thus, the retron-Eco9 encodes a TA system similar to retron-Sen2, and its toxin (RcaT-Eco9) is inhibited by a retronencoded antitoxin (RT-msDNA-Eco9).

To assess which part of the antitoxin unit is cognate to the toxin, we constructed chimeric retron constructs between Sen2 and Eco9. Retron-RTs are known to be highly specific in reverse transcribing their cognate msrmsd-RNA, by binding to specific RNA-structures of the msr region ${ }^{25}$. Cross-specificity between non-cognate RT-msrmsd pairs has only been observed between highly homologous retrons ${ }^{13}$. Thus, we first evaluated if the Sen2 and Eco9 RTs could transcribe their non-cognate msrmsd. To assess this, we isolated msDNA from $E$. coli co-expressing PBAD-RT plasmids (Sen2 [Se], or Eco9 [Ec]) with Ptac-msrmsd plasmids (Se, Ec, or -). Both RT-Sen2 and RT-Eco9 could use their non-cognate msrmsd and produce msDNA (Fig. 4A), albeit RT-Eco9 was slightly less efficient (lane Ec-Se - see also ED. Fig 10F for repercussions of this lower efficiency).

To assess the specificity of RcaT to RT, we then made arabinose-inducible plasmids, carrying binary combinations of both RcaT and RT from retrons Se, and Ec: Se-Se, Ec-Se, Se-Ec, and Ec-Ec. We co-expressed these plasmids with IPTG-inducible Ptac plasmids, expressing msrmsd from Sen2, Eco9, or the empty vector, to test which of the RT-msDNA systems retained the antitoxin-activity. As expected, both Sen2 and Eco9 RcaT inhibited the growth of E. coli in the absence of msDNA, irrespectively of the RT co-expressed (Fig. 4B). While cognate or non-cognate msDNA template could activate the antitoxin activity in cognate RTRcaT combinations (Se-Se, or Ec-Ec) and neutralize the RcaT toxicity, this did not work in non-cognate RT-RcaT combinations (Se-Ec, or Ec-Se) (Fig. 4B, ED Fig 10F). This demonstrates that although RTs can produce msDNA from non-cognate msrmsd templates, 
217 and the formed RT-msDNA complex is an active antitoxin, this active antitoxin can only act 218 against its cognate toxin. Thus, the RT-RcaT interactions (Fig. 3) are cognate within retron-

219 TAs, and essential for antitoxin-activity.

220

221 In summary, the RT-RcaT interaction provides the specificity for the TA system, as the RT can 222 interact directly with RcaT in the absence of msDNA, and it cannot be exchanged for a 223 homologous RT from another retron. In contrast, the msDNA sequence is interchangeable, at 224 least to some extent, but the RT-msDNA interaction is absolutely required to form an active 225 antitoxin unit.

\section{DISCUSSION}

High-throughput reverse-genetics approaches have revolutionized the characterization of gene function in bacteria ${ }^{26,27}$, providing rich phenotypic information and genetic links that can be used to identify the enigmatic function of fully uncharacterized genes or to further our understanding of known cellular processes ${ }^{28-30}$. Here, we have used the gene-deletion phenotypes related to the retron-Sen2 to show that retrons encode a novel family of tripartite TA systems. In retrons, the accessory gene is a toxin (RcaT) and the RT-msDNA form together an antitoxin complex (Fig. 5).

There are a number of intriguing aspects of this new tripartite TA family that we have resolved. First, the RT is the specificity determinant of the antitoxin, by interacting with RcaT. However, alone, the RT does not affect RcaT toxicity, as the RcaT-RT complex is an active toxin (Fig. 1D, Fig. 4A), even though the RT and RcaT are tightly bound in all the conditions we tested (Fig. 3). Second, it is the processed msDNA that activates the RT-msDNA antitoxin, rather than the msrmsd-RNA or intermediate msDNA-biosynthesis forms. This finding is corroborated by the fact that when msDNA biosynthesis is stopped at steps where intermediate products become stable, then the RcaT toxin becomes active (point mutation in the branching $\mathrm{G}$ of $\mathrm{msr}$ stabilizing the msrmsd-RNA [ED Fig. 4A] ${ }^{11}$, and $x s e A / x \operatorname{se} B$ mutations stabilizing the unprocessed RNA-DNA hybrid [Fig. 1D, ED Fig. 4C]). Third, msDNA is required, but is not sufficient for antitoxin activity. This last point is harder to establish, as mSDNA is not produced in cells lacking its cognate RT. We bypassed this limitation by using a second retron, to make hybrid-retrons. Although non-cognate RT-msrmsd pairs produce msDNA (Fig. 4A), and these form active antitoxins against cognate RT-RcaT pairs, they were not enough to inhibit the noncognate RT-RcaT pairs (Fig. 4B). Therefore, RTs are needed for the antitoxin activity, not only

251 because they produce msDNA, but also because they provide the antitoxin specificity by simultaneously binding the toxin (Fig. 3) and the msDNA (ED Fig. 9C). 
There are also several open aspects of this retron-TA model. Although we provide strong evidence that the RT-msDNA interaction activates the antitoxin unit, the exact mechanism remains to be resolved. It is still possible that the msDNA also interacts with RcaT in the tripartite RcaT-RT-msDNA complex. In addition, we do not know the cellular target of RcaT, or why it preferentially inhibits growth in cold and anaerobic conditions, and only upon overexpression it impacts growth at $37^{\circ} \mathrm{C}$ in aerobic conditions. The two phenotypes may be linked, due to the target of the toxin being more relevant in cold/anaerobiosis. Alternatively, the toxin itself could be post-translationally activated in these conditions. A very different toxin in Pseudomonas putida, GraT, also causes cold-sensitivity upon antitoxin perturbations, and toxicity when over-expressed ${ }^{31}$. GraT seems to cause cold-sensitivity by attacking ribosome biogenesis ${ }^{32}$. Yet, an anaerobic-sensitivity toxin phenotype has not been reported before for GraT, or any toxin of any TA system as far as we know. Therefore, it is possible that RcaT has a distinct toxicity mechanism. Finally, we show here that another retron in E. coli NILS-16 encodes a similar TA system, but there are myriads of retrons, out of which only a few dozen have been experimentally verified ${ }^{4}$. These carry diverse retron-components and accessory genes, and it remains to be seen if all or only a subset of them encode TA systems.

TA systems are usually bipartite, and are divided in four major types, based on how the antitoxin neutralizes the toxin ${ }^{20}$. Retron-TAs are similar to type II systems, since the antitoxin (RT-msDNA) seems to inhibit RcaT through a direct protein-protein interaction. Yet, they form a distinct family of tripartite TA systems, since the msDNA is absolutely required for the antitoxin function. A number of other housekeeping genes are also required for producing the mature msDNA, increasing the complexity and the dependencies of this TA system. The evolutionary advantage of retaining such complex selfish systems is hard to fathom, as the physiological role of most TA systems remains largely unknown. In the accompanying paper ${ }^{33}$, we provide an insight into this question. The exact complexity of this TA system and its msDNA component seem to allow retrons to directly sense different phage functionalities, and hence protect bacteria from phage attack.

\section{ACKNOWLEDGEMENTS}

284 We thank the EMBL Genomics Core Facility, and especially Anja Telzerow and Vladimir Benes for preparing the whole-genome sequencing; the EMBL Protein Expression \& Purification Core Facility, and especially Jacob Scheurich and Kim Remans for purifying protein RT-Sen2; Nazgul Sakenova for help for identification of retron-Eco9, and all members of the Typas lab

288 for discussions. This work was supported by the European Molecular Biology Laboratory and 289 the Sofja Kovaleskaja Award of the Alexander von Humboldt Foundation. AM was supported 290 by a fellowship from the EMBL Interdisciplinary Postdoc (EI3POD) programme under Marie 
291 Skłodowska-Curie Actions COFUND (grant number 664726). JRE was supported by the 292 National Institutes of Health (K08AI108794). HAP is supported by NIFA (NIFA 2016-11004 \& 293 2017-08881) and DARPA. AT is supported by an ERC consolidator grant, uCARE.

\section{AUTHOR CONTRIBUTIONS}

296 MMS, JRE, HAP, \& AT supervised the study. JB, JRE, HAP, \& AT conceived this study. JB 297 and AT designed the experiments, and JB, AM, SGS, and CK performed them. The STm 298 chemical-genetics screen data is from BP \& MG. MG analyzed the sequencing data and FS 299 the AP-qMS data. JB \& AM designed figures, with inputs from AT. JB \& AT wrote the 300 manuscript with input from all authors.

\section{DATA AVAILABILITY STATEMENT}

303 Raw reads from whole genome sequencings are available at the European Nucleotide Archive, 304 under project number PRJEB38324. Proteomics data from protein affinity purifications can be 305 found in Table S1, and raw data will be uploaded in an appropriate server prior to publication. All unprocessed source images are available upon request.

\section{COMPETING INTEREST DECLARATION}

309 We declare no competing financial interests.

\section{ADDITIONAL INFORMATION}

312 Supplementary information is available for this paper. Correspondence and requests for 313 materials should be addressed to AT (typas@embl.de). 


\section{FIGURE LEGENDS}

315 Figure 1. STm Retron-Sen2 deletion mutants are cold-sensitive.

316 (A) Genes involved in msDNA-Sen2 biosynthesis. Retron-Sen2 is an operon containing 317 msrmsd (msrmsd-RNA), STM14_4640 (STM3845), and rrtT (STM14_4641; STM3846; RT318 Sen2). $\quad r n h A$ (coding for Ribonuclease $H$ ) and $x \operatorname{se} A / x \operatorname{se} B$ (coding together for 319 Exodeoxyribonuclease VII - Exo VII) are not genetically linked to the Retron-Sen2, or to each 320 other.

321 (B) Pathway of msDNA-Sen2 production. RT-Sen2 binds to msr-RNA and reverse transcribes 322 msd-RNA to msd-DNA, while ribonuclease $\mathrm{H}(\mathrm{RN}$ ase $\mathrm{H})$ degrades the msd-RNA template. 323 msr-RNA and msd-DNA are joined by a 2'-5' phosphodiester bond (RNA-DNA 2'-5'), and by 324 RNA-DNA hydrogen bonds at the ends of msr-RNA/msd-DNA. Exo VII cleaves the first four 325 nucleotides off the start of the msd-DNA, separating the DNA/RNA hybrid into msDNA-Sen2 326 and msr-Sen2. msDNA-Sen2 remains complexed with RT-Sen2.

327 (C) Strains $\Delta r r t T$ and $\triangle x s e A$ are cold-sensitive. 1536 colony-arrays of the STm gene-deletion 328 library ${ }^{22}$ were pinned onto LB plates, and strains were grown at room temperature. Colony sizes ${ }^{34}$ were used to calculate a fitness S-score for each strain ${ }^{35}$. S-scores were calculated from $n=8$. Dashed vertical line denotes the mean fitness S-score calculated from all strains ( $n=3781)$; negative and positive S-scores indicate sensitive and resistant mutants, respectively.

333 (D) Perturbing msDNA-biogenesis leads to cold sensitivity. STm retron-deletion strains were grown for 5-6 hours at $37^{\circ} \mathrm{C}$ in LB, serially diluted, and spotted on LB plates. Plates were incubated either at $15^{\circ} \mathrm{C}$ and $37^{\circ} \mathrm{C}$. Representative data shown from four independent experiments.

Figure 2. RcaT is a toxin that causes the retron phenotypes.

(A) Cold-sensitivity suppressor mutations map almost exclusively in rcaT. Suppressors from STm strains ( $\Delta r r t T, \Delta x s e A, \Delta m s r m s d$ ) were isolated from LB plates incubated at $15^{\circ} \mathrm{C}$, their genomic DNA was sequenced and compared to that of wildtype STm to map the mutations. (B) Deleting rcaT (STM14_4640) reverts the cold-sensitivity of retron mutants. STm strains were grown for $5-6$ hours at $37^{\circ} \mathrm{C}$ in LB, serially diluted, and spotted on LB plates. Plates were

344 incubated either at $15^{\circ} \mathrm{C}$ or $37^{\circ} \mathrm{C}$. Representative data shown from two independent 345 experiments.

346 (C) Deleting rcaT reverts the anaerobic sensitivity of retron mutants. Growth curves of STm 347 strains were obtained from measuring OD 578 in microplates under anaerobic conditions at $34837^{\circ} \mathrm{C}$. Each point is the average optical density (OD595) of $n=11$ (technical replicates), error 349 bars denote standard deviation (error bars not shown if smaller than symbols). 
(D) Overexpressing rcaT is toxic in E. coli at $37^{\circ} \mathrm{C}$. E. coli BL21 Arabinose-inducible (BL21-AI) carrying plasmids $\mathrm{p}$-rcaT, or p-rcaT-D296V, or an empty vector (p-empty) were grown for 5-6 hours at $37^{\circ} \mathrm{C}$ in kanamycin-LB, serially diluted, spotted on kanamycin-LB plates with or without arabinose, and then incubated overnight at $37^{\circ} \mathrm{C}$. Representative data shown from two independent experiments.

Figure 3. RT and RcaT reciprocally co-immunoprecipitate independent of the presence of msDNA or temperature Immunoprecipitation of RT-3xFLAG (A-B) and RcaT-3xFLAG (C-D) at $37^{\circ} \mathrm{C}$ (A, C) and $20^{\circ} \mathrm{C}$ (B, D). Chromosomally tagged rrtT-3xFlag or rcaT-3xFlag STm strains (WT, $\Delta x s e A, \Delta m s r m s d$ ) grown at $37^{\circ} \mathrm{C}$ (and for $\mathbf{B}, \mathbf{D}$ shifted for 5 hours at $20^{\circ} \mathrm{C}$ ) were used for AP-qMS. Protein abundances of IP samples of the strains carrying the tagged protein are compared to IP samples of untagged STm (y-axis). Data shown are the average from two biological replicates.

Figure 4. RT-RcaT interactions determine the specificity of the RT-msDNA antitoxin.

(A) Non-cognate RT-msrmsd pairs produce msDNA. msDNA were extracted from E. coli BW25113 co-expressing RT of retron-Sen2 (Se) or -Eco9 (Ec) (PBAD-RT; Se, or Ec-arabinose induction), and plasmids carrying msrmsd (Ptac-msrmsd; Se, or Ec - IPTG induction), or an empty vector (-). Extracted msDNA were electrophoresed in a TBE-Polyacrylamide gel. Representative data shown from two independent experiments.

(B) RcaT toxicity is inhibited by the cognate RT loaded with msDNA - cognate or not. E. coli BW25113 was co-transformed with plasmids carrying RT-RcaT combinations of retron-Sen2 (Se) and -Eco9 (Ec) (PBAD-RT-RcaT; Se-Se, Se-Ec, Ec-Se, or Ec-Ec - arabinose induction), and plasmids carrying msrmsd (Ptac-msrmsd; Se, or Ec - IPTG induction) or an empty vector (-). Strains were grown for $5-6$ hours at $37^{\circ} \mathrm{C}$ in LB with appropriate antibiotics, serially diluted, spotted on LB plates with IPTG, antibiotics, and arabinose (+/-), and then incubated overnight at $37^{\circ} \mathrm{C}$. Representative data shown from two independent experiments.

Figure 5. Model of retron-TA mode of action. RT-msDNA complex (antitoxin) inactivates RcaT (toxin) by direct binding. RT (blue) produces and binds msDNA (orange). The RT binds also to RcaT. Only the active antitoxin complex RT-msDNA can counterbalance the toxic activity of RcaT (light purple). RT provides the antitoxin specificity towards RcaT, but alone cannot inactive the toxin. Perturbing msDNA biosynthesis disrupts the RT-msDNA antitoxin complex, allowing for RcaT (purple) to act as toxin alone, or bound to RT. 
bioRxiv preprint doi: https://doi.org/10.1101/2020.06.22.160168; this version posted June 22, 2020. The copyright holder for this preprint (which was not certified by peer review) is the author/funder, who has granted bioRxiv a license to display the preprint in perpetuity. It is made available under aCC-BY 4.0 International license.

\section{REFERENCES}

1. Lampson, B. C., Inouye, M. \& Inouye, S. Reverse transcriptase with concomitant ribonuclease $\mathrm{H}$ activity in the cell-free synthesis of branched RNA-linked msDNA of Myxococcus xanthus. Cell 56, 701-707 (1989).

2. Lampson, B. C. et al. Reverse transcriptase in a clinical strain of escherichia coli: Production of branched RNA-linked msDNA. Science (80-. ). 243, 1033-1038 (1989).

3. Yee, T., Furuichi, T., Inouye, S. \& Inouye, M. Multicopy single-stranded DNA isolated from a gramnegative bacterium, Myxococcus xanthus. Cell 38, 203-209 (1984).

4. Simon, A. J., Ellington, A. D. \& Finkelstein, I. J. Retrons and their applications in genome engineering. Nucleic Acids Res. 47, 11007-11019 (2019).

5. Farzadfard, F. \& Lu, T. K. Genomically encoded analog memory with precise in vivo DNA writing in living cell populations. Science (80-. ). 346, 1256272-1256272 (2014).

6. Simon, A. J., Morrow, B. R. \& Ellington, A. D. Retroelement-Based Genome Editing and Evolution. ACS Synth. Biol. 7, 2600-2611 (2018).

Farzadfard, A. F. et al. Efficient Retroelement-Mediated DNA Writing in Bacteria. Preprint at https://doi.org/10.1101/2020.02.21.958983 (2020).

8. Schubert, M. G. et al. High throughput functional variant screens via in-vivo production of single-stranded DNA. Preprint at https://doi.org/10.1101/2020.03.05.975441 (2020).

9. Inouye, K., Tanimoto, S., Kamimoto, M., Shimamoto, T. \& Shimamoto, T. Two novel retron elements are replaced with retron-Vc95 in Vibrio cholerae. Microbiol. Immunol. 55, 510-513 (2011).

10. Dhundale, A., Lampson, B., Furuichi, T., Inouye, M. \& Inouye, S. Structure of msDNA from myxococcus xanthus: Evidence for a long, self-annealing RNA precursor for the covalently linked, branched RNA. Cell 51, 1105-1112 (1987).

11. Hsu, M. Y., Inouye, S. \& Inouye, M. Structural requirements of the RNA precursor for the biosynthesis of the branched RNA-linked multicopy single-stranded DNA of Myxococcus xanthus. J. Biol. Chem. 264, 6214-6219 (1989).

Lim, D. Structure and biosynthesis of unbranched multicopy single-stranded DNA by reverse

13. Lima, T. M. O. \& Lim, D. A novel retron that produces RNA-less msDNA in Escherichia coli using reverse transcriptase. Plasmid 38, 25-33 (1997).

14. Jung, H., Liang, J., Jung, Y. \& Lim, D. Characterization of cell death in Escherichia coli mediated by XseA, a large subunit of exonuclease VII. J. Microbiol. 53, 820-828 (2015).

15. Lampson, B. C., Viswanathan, M., Inouye, M. \& Inouye, S. Reverse transcriptase from Escherichia coli exists as a complex with msDNA and is able to synthesize double-stranded DNA. J. Biol. Chem. 265, 8490-8496 (1990).

16. Jeong, D. W., Kim, K. \& Lim, D. Evidence for the Complex Formation between Reverse Transcriptase and Multicopy Single-stranded DNA in Retron EC83. Mol. Cells 7, 347-351 (1997).

17. Ahmed, A. M. \& Shimamoto, T. msDNA-St85, a multicopy single-stranded DNA isolated from Salmonella enterica serovar Typhimurium LT2 with the genomic analysis of its retron. FEMS Microbiol. Lett. 224, 291-297 (2003).

18. Elfenbein, J. R. et al. Multicopy Single-Stranded DNA Directs Intestinal Colonization of Enteric Pathogens. PLoS Genet. 11, 1-24 (2015).

19. Elfenbein, J. R. et al. Novel determinants of intestinal colonization of salmonella enterica serotype typhimurium identified in bovine enteric infection. Infect. Immun. 81, 4311-4320 (2013).

20. Harms, A., Brodersen, D. E., Mitarai, N. \& Gerdes, K. Toxins, Targets, and Triggers: An Overview of

21. Bleibtreu, A. et al. The rpoS Gene Is Predominantly Inactivated during Laboratory Storage and Undergoes Source-Sink Evolution in Escherichia coli Species. J. Bacteriol. 196, 4276-4284 (2014). 
bioRxiv preprint doi: https://doi org/10.1101/2020.06.22 160168; this version posted June 22, 2020. The copyright holder for this preprint (which was not certified by peer review) is the author/funder, who has granted bioRxiv a license to display the preprint in perpetuity. It is made available under aCC-BY 4.0 International license.

23. Hall, A. M., Gollan, B. \& Helaine, S. Toxin-antitoxin systems: reversible toxicity. Curr. Opin. Microbiol. 36, 102-110 (2017).

24. Galardini, M. et al. Phenotype inference in an Escherichia coli strain panel. Elife 6, (2017).

25. Inouye, S., Hsu, M. Y., Xu, A. \& Inouye, M. Highly specific recognition of primer RNA structures for 2'-OH priming reaction by bacterial reverse transcriptases. J. Biol. Chem. 274, 31236-31244 (1999).

440 26. Nichols, R. J. et al. Phenotypic Landscape of a Bacterial Cell. Cell 144, 143-156 (2011).

27. Price, M. N. et al. Mutant phenotypes for thousands of bacterial genes of unknown function. Nature 557, 503-509 (2018).

28. Typas, A. et al. Regulation of Peptidoglycan Synthesis by Outer-Membrane Proteins. Cell 143, 10971109 (2010).

29. Paradis-Bleau, C., Kritikos, G., Orlova, K., Typas, A. \& Bernhardt, T. G. A Genome-Wide Screen for Bacterial Envelope Biogenesis Mutants Identifies a Novel Factor Involved in Cell Wall Precursor Metabolism. PLoS Genet. 10, e1004056 (2014).

30. Gray, A. N. et al. Coordination of peptidoglycan synthesis and outer membrane constriction during Escherichia coli cell division. Elife 4, 1-29 (2015).

31. Tamman, H., Ainelo, A., Ainsaar, K. \& Hõrak, R. A moderate toxin, grat, modulates growth rate and stress tolerance of pseudomonas putida. J. Bacteriol. 196, 157-169 (2014).

32. Ainelo, A., Tamman, H., Leppik, M., Remme, J. \& Hõrak, R. The toxin GraT inhibits ribosome biogenesis. Mol. Microbiol. 100, 719-734 (2016).

33. Bobonis, J. et al. Phage proteins block and trigger retron toxin/antitoxin systems. Preprint at bioRxiv (2020).

34. Kritikos, G. et al. A tool named Iris for versatile high-throughput phenotyping in microorganisms. Nat. Microbiol. 2, 17014 (2017). 
461

462

463

464

465

466

467

468

469

470

471

472

473

474

475

476

477

478

479

480

481

482

483

484

485

486

487

488

489

490

491

492

493

494

495

496

\section{METHODS}

\section{Bacterial strains, plasmids, primers, and growth conditions}

Genotypes of bacterial strains, plasmid description/construction strategy, and sequences of primers used in this study are listed in Tables S2-S5, respectively. Bacteria were grown in Lysogeny Broth Lennox (LB-Lennox; Tryptone $10 \mathrm{~g} / \mathrm{L}$, Yeast Extract $5 \mathrm{~g} / \mathrm{L}$, Sodium Chloride 5 g/L). LB-Agar plates (LB plates) were prepared by adding separately autoclaved $2 \%$ moltenAgar in liquid LB. All plasmid-carrying bacterial strains were streaked-out/grown/assayed with appropriate antibiotics, in order to maintain the plasmids. Plasmids carrying PBAD-inserts were induced with $0.2 \% D$-arabinose. Plasmids carrying Ptac-inserts were induced with $0.1 \mathrm{mM}$ Isopropyl $\beta$ - d-1-thiogalactopyranoside (IPTG). Bacterial strains with chromosomally-inserted antibiotic resistance cassettes were streaked out from stocks on antibiotic-LB plates, but grown/assayed thereafter without antibiotics. Antibiotics used were Kanamycin $(30 \mu \mathrm{g} / \mathrm{mL})$, Ampicillin $(50 \mu \mathrm{g} / \mathrm{mL})$, Spectinomycin $(100 \mu \mathrm{g} / \mathrm{mL})$ and Chloramphenicol $(20 \mu \mathrm{g} / \mathrm{mL})$. Coldsensitive strains (STm retron-Sen2 mutants) were freshly streaked-out from glycerol stocks and kept only at $37^{\circ} \mathrm{C}$ before every experiment, in order to avoid suppressor mutations.

\section{Genetic techniques}

Salmonella enterica subsp. enterica ser. Typhimurium str. 14028s (STm) chromosomal deletion strains were acquired from the STm single-gene deletion library ${ }^{22}$. STm strains $\triangle r n h A$, $\triangle x s e B, \triangle m s r m s d$, and $\triangle a r a B A D$ were constructed through $\lambda$-red recombineering ${ }^{36}$, with primer-design for deletions as described in ${ }^{37}$. Escherichia coli strains WT (BW25113), $\Delta x s e A$, $\Delta x s e B$, and $\Delta r n h A$ were acquired from the Keio collection ${ }^{37}$. All single-gene deletion strains, newly constructed and from libraries, were re-transduced in wildtype STm or E. coli strains by P22 and P1 transduction, respectively. Resistance cassettes from single-gene deletions were flipped-out using the yeast-flippase expressing-plasmid, pCP20 ${ }^{38}$. To make double-gene deletion strains, antibiotic resistance cassette genetic deletions were transduced in deletion strains in which the first marker was already flipped out. $\Delta m s d$ deletion strains (ED Fig. $2 \mathrm{~B}$, 2C, 2D) were made in two stages. First, scar-less deletions in the msd region were constructed on a plasmid-vector, either by amplifying and cloning msd deletions from the corresponding suppressors (ED Fig. 2A), or by PCR-based directed-deletions on a plasmid carrying msrmsd 39. Second, the constructed msd deletion-fragments were amplified, and replaced the chromosomal-WT msrmsd locus by ccdB-recombineering ${ }^{40}$ (see tables S3-S4 for details on plasmids and primers used). Plasmids were transformed in E. coli BW25113 strains by TSS ${ }^{41}$, while E. coli BL21 and STm strains were transformed by electroporation ${ }^{42}$. 
For measuring growth curves anaerobically, LB was pre-reduced in anoxic conditions in an anaerobic chamber $\left(2 \% \mathrm{H}_{2}, 12 \% \mathrm{CO}_{2}, 86 \% \mathrm{~N}_{2}\right.$; Coy Laboratory Products) for two days before use. Flat-bottomed transparent 96-well plates containing $90 \mu \mathrm{L}$ LB were inoculated with STm strains (grown aerobically overnight at $37^{\circ} \mathrm{C}$ ) at $\mathrm{OD}_{595}=0.01$, and sealed with breathable membranes (Breathe-Easy). Plates were incubated at $37^{\circ} \mathrm{C}$ in the anaerobic chamber (without shaking), and OD578 was measured periodically (EON Biotek microplate spectrophotometer). For measuring growth under aerobic conditions at $37^{\circ} \mathrm{C}$, plates were instead incubated with shaking (200 rpm), and OD578 was measured (Tecan Safire2 microplate spectrophotometer).

For the growth curves at $15^{\circ} \mathrm{C}$, overnight cultures were inoculated in flasks of LB (OD $\left.595=0.01\right)$ and grown in a refrigerated incubator (Infors Multitron HT) with shaking (180 rpm). Growth was monitored by measuring $\mathrm{OD}_{595}$. For the viability tests samples were periodically taken, serially diluted, and plated on LB-plates. Colony Forming Units per culture volume $(\mathrm{CFU} / \mathrm{mL})$ were calculated after overnight growth at $37^{\circ} \mathrm{C}$. For the viability curves in ED Fig. 3D, E. coli p-empty and p-rcaT strains were inoculated in $L B\left(O_{595}=0.01\right)$, and cultures were incubated until $\mathrm{OD}_{595}=0.4$ at $37^{\circ} \mathrm{C}$ with shaking $(180 \mathrm{rpm})$. Subsequently, cultures were transferred at $15^{\circ} \mathrm{C}$ with shaking $(180 \mathrm{rpm})$ for $30 \mathrm{~min}$. Plasmids were then induced with $0.2 \%$ arabinose, and viability was monitored by periodically plating culture-samples on ampicillin LB-plates.

\section{Spot growth tests}

517 Single bacterial-colonies were inoculated in $2 \mathrm{~mL} \mathrm{LB}$, and incubated over-day aerobically at $51837^{\circ} \mathrm{C}$ in a roller drum (6 hours, until OD $\left.595 \sim 5\right)$. Over-day cultures were stepwise serially-diluted eight times (ten-fold) in LB $(100 \mu \mathrm{L}$ culture $+900 \mu \mathrm{L}$ LB). Using a 96-pinner (V\&P Scientific, catalogue number: VP 404), $\sim 10 \mu \mathrm{L}$ of culture dilutions were spotted on LB-plates containing appropriate antibiotics, and $0.2 \%$ arabinose if needed (arabinose was only present in plates, not in cultures). Spots in growth tests shown in Figures 1D, 2D, ED 1A, ED 2A, ED 3C, and ED 9B were spotted manually ( $10 \mu \mathrm{L})$ with a multichannel pipette. LB-plates were incubated overnight (13-15 hours) aerobically at $37^{\circ} \mathrm{C}$, in a humid incubator. For cold-sensitivity growth tests, LB-plates were incubated for 36,48 , or 72 hours, at $25^{\circ} \mathrm{C}, 20^{\circ} \mathrm{C}$, or $15^{\circ} \mathrm{C}$, respectively.

\section{msDNA isolation and running msDNA in TBE-Acrylamide gels}

msDNA was isolated by alkaline lysis (reagents as described in ${ }^{43}$ ). msDNA was over-produced by over-expressing the reverse transcriptase and the msrmsd region (msrmsd-rrtT), in order to be able to purify msDNA from small culture volumes (see Tables S2-S3 for strain/plasmid combinations used for each msDNA isolation). Strains were inoculated at OD595=0.01 in 20

$532 \mathrm{~mL}$ LB, supplemented with appropriate antibiotics and $0.2 \%$ arabinose (to over-express $533 \mathrm{msDNA}$ ). Cultures were incubated for $5-6$ hours at $37^{\circ} \mathrm{C}$ with rigorous shaking. After this, cells 
534 were put on ice and approximately $10 \mathrm{~mL}$ was centrifuged $\left(4,000 \mathrm{rpm} / 15 \mathrm{~min} / 4^{\circ} \mathrm{C}\right)$ - after 535 correcting for $\mathrm{OD}_{595}$. Pellets were washed once with ice-cold PBS, re-suspended in alkaline

536 solution 1, transferred into $1.5 \mathrm{~mL}$ Eppendorf tubes, and alkaline solutions II and III were cycled 537 as described in ${ }^{43}$. After centrifugation $\left(14,000 \mathrm{rpm} / 20 \mathrm{~min} / 4^{\circ} \mathrm{C}\right)$, supernatants were extracted 538 twice with Phenol: Chloroform: Isoamyl-Alcohol (50:48:2, pH 8), and nucleic acids were 539 precipitated overnight at $4^{\circ} \mathrm{C}$ with isopropanol. Precipitated nucleic acids were centrifuged at $54014,000 \mathrm{rpm} / 60 \mathrm{~min} / 4^{\circ} \mathrm{C}$. Pellets were re-suspended once with $1 \mathrm{~mL}$ of $70 \%$ Ethanol, and 541 centrifuged again at $14,000 \mathrm{rpm} / 60 \mathrm{~min} / 4^{\circ} \mathrm{C}$. Pellets (msDNA extracts) were air-dried (15 542 minutes), resuspended in $10 \mu \mathrm{L}$ of distilled water containing RNase $A(20 \mu \mathrm{g} / \mathrm{mL})$, and 543 incubated at $37^{\circ} \mathrm{C}$ for 30 minutes. Samples were subsequently kept at $-80^{\circ} \mathrm{C}$ until further use. 544 msDNA extracts $(10 \mu \mathrm{L}$ ) were electrophoresed (70 Volts, 3.5 hours) in 1x-TBE:12\%545 Polyacrylamide gels (with 1x-TBE buffer), and stained with ethidium bromide. 50 bp ladder 546 was from Promega (catalogue No. G4521).

\section{Affinity purifications (APs)}

549 Two biological replicates of STm Flag-tagged strains (and appropriate negative controls, i.e., strains with the same genetic background but in which no gene was tagged) were inoculated in $100 \mathrm{~mL}$ LB (starting OD $595=0.02$ ), and grown at $37^{\circ} \mathrm{C}$ with constant shaking (180 rpm), until OD595 $=1.2-1.5$. Cultures were split in half, and one flask was transferred at $20^{\circ} \mathrm{C}$ with constant shaking (180 rpm) for 5 hours. The remaining volume was used to prepare the $37^{\circ} \mathrm{C}$ samples. From this stage on, samples were kept on ice. Approximately $50 \mathrm{~mL} / \mathrm{OD}_{595}=1.5$ of cultures were transferred to $50 \mathrm{~mL}$ tubes, with culture volumes per strain being normalized based on OD to adjust for total protein-levels across strains. Cultures were centrifuged at 5,000 $\mathrm{rpm} / 10 \mathrm{~min} / 4^{\circ} \mathrm{C}$ and the supernatant was discarded. Pellets were washed once with $50 \mathrm{~mL}$ of ice-cold PBS, and cells were centrifuged again. Pellets were then frozen at $-80^{\circ} \mathrm{C}$. Subsequently, pellets were re-suspended in $1.2 \mathrm{~mL}$ of Lysis Buffer $(50 \mu \mathrm{g} / \mathrm{ml}$ lysozyme, $0.8 \%$ NP-40, $1 \mathrm{mM} \mathrm{MgCl}$ 2, $1 x$ protease inhibitors [Roche; cOmplete Protease Inhibitor Cocktail] in PBS), and transferred to Eppendorf tubes. Cells were lysed by ten freeze-thawing cycles (frozen in liquid nitrogen, thawing 5 minutes at $25^{\circ} \mathrm{C}$ with $1,400 \mathrm{rpm}$ shaking per cycle). Lysates were centrifuged at $14,000 \mathrm{rpm} / 60 \mathrm{~min} / 4^{\circ} \mathrm{C}$ to remove intact cells and other insoluble components. Samples were taken at this step (input samples). Flag-beads (ANTI-FLAG® M2 Affinity Agarose Gel; Sigma-Aldrich) were washed twice (20x the beads volume) with Wash Buffer ( $0.8 \%$ NP-40 in PBS), and $25 \mu \mathrm{L}$ of washed Flag-beads were added to $\sim 1 \mathrm{~mL}$ of lysate.

567 Lysates were incubated with Flag-beads overnight on a table-top roller, at $4^{\circ} \mathrm{C}$. Subsequently, 568 beads were centrifuged at $8,200 \mathrm{rcf} / 10 \mathrm{~min} / \mathrm{RT}$, and the supernatants were discarded. The 569 beads were washed four times with $1 \mathrm{~mL}$ of Wash Buffer (2 min rolling with table-top roller and 570 centrifuged at $8,200 \mathrm{rcf} / 2 \mathrm{~min} / \mathrm{RT}$ per wash cycle). After the final wash, $50 \mu \mathrm{L}$ of Elution Buffer 
$571 \quad(150 \mu \mathrm{g} / \mathrm{mL}$ 3xFlag peptide [Sigma-Aldrich], 0.05\% Rapigest [Waters], 1x protease inhibitors 572 in PBS) was added to the Flag-beads, and proteins were eluted for 2 hours on a table-top

573 roller, at $4^{\circ} \mathrm{C}$. The samples were centrifuged at $8,200 \mathrm{rcf} / 15 \mathrm{~min} / \mathrm{RT}, 50 \mu \mathrm{L}$ of eluates were

574 retrieved, and transferred to Eppendorf tubes (IP samples).

\section{Proteomics analysis of APs}

577 Proteins were digested according to a modified SP3 protocol ${ }^{44}$. Briefly, approximately $2 \mu \mathrm{g}$ of protein was diluted to a total volume of $20 \mu \mathrm{L}$ of water and added to the bead suspension (10 $\mu \mathrm{g}$ of beads (Thermo Fischer Scientific_Sera-Mag Speed Beads, CAT\# 4515-2105-050250,

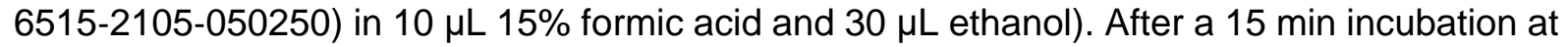
room temperature with shaking, beads were washed four times with $70 \%$ ethanol. Next, proteins were digested overnight by adding $40 \mu \mathrm{L}$ of digest solution ( $5 \mathrm{mM}$ chloroacetamide, $1.25 \mathrm{mM}$ TCEP, $200 \mathrm{ng}$ trypsin, and $200 \mathrm{ng}$ LysC in $100 \mathrm{mM}$ HEPES pH 8). Peptides were eluted from the beads, dried under vacuum, reconstituted in $10 \mu \mathrm{L}$ of water, and labelled for $30 \mathrm{~min}$ at room temperature with $17 \mu \mathrm{g}$ of TMT10plex (Thermo Fisher Scientific) dissolved in experiments belonging to the same mass spectrometry run were combined. Samples were desalted with solid-phase extraction on a Waters OASIS HLB $\mu$ Elution Plate $(30 \mu \mathrm{m})$ and fractionated under high $\mathrm{pH}$ conditions prior to analysis with liquid chromatography coupled to tandem mass spectrometry ( $Q$ Exactive Plus; Thermo Fisher Scientific), as previously described ${ }^{45}$. Mass spectrometry raw files were processed with isobarQuant, and peptide and protein identification was performed with Mascot 2.5.1 (Matrix Science) against the STm UniProt FASTA (Proteome ID: UP000001014), modified to include known contaminants and the reversed protein sequences (search parameters: trypsin; missed cleavages 3; peptide tolerance $10 \mathrm{ppm}$; MS/MS tolerance $0.02 \mathrm{Da}$; fixed modifications were carbamidomethyl on cysteines and TMT10plex on lysine; variable modifications included acetylation on protein $\mathrm{N}$ terminus, oxidation of methionine, and TMT10plex on peptide $\mathrm{N}$-termini).

The fold-enrichment of pulled-down proteins in Flag-tagged strains compared to negative controls was calculated, and statistical significance was evaluated using limma analysis ${ }^{46}$. A similar analysis was conducted on the input samples to ensure that enriched proteins were not overexpressed in Flag-tagged strains.

\section{Whole genome sequencing}

605 Genomic DNAs from retron-mutant suppressor strains were isolated using a kit, by following 
608

609

610

611

612

613

614

615

616

617

618

619

620

621

622

623

624

625

626

627

628

629

630

631

632

633

634

635

636

637

638

639

640

641

642

643

644

with sonication for 2 minutes, and libraries were constructed using a kit (NEB Ultra DNA library kit for Illumina; catalogue number E7370L), according to the manufacturer's instructions. The 30 genomic libraries were sequenced using a NextSeq Illumina platform with a 150 base pairs paired-end configuration. Variants were called using breseq v0.28.0 ${ }^{47}$, using the Salmonella enterica subsp. enterica serovar Typhimurium strain 14028S genome as reference (RefSeq ID: NC_016856.1). Genotypes of suppressor strains can be found in Table S2.

\section{RT-Sen2 purification and DNA-isolation from RT-Sen2 purified protein}

Plasmid pJB120 (pET28a-msrmsd-rrtT-His; described in Tables S3-S4) was used to overexpress a C-terminally His-tagged RT-Sen2 protein, along with msrmsd-RNA. Strain E. coli BL21 (DE3) CodonPlus-RIL was electroporated with pJB120. An overnight culture of the overexpression strain was inoculated at $\mathrm{OD}_{595}=0.01$ in $100 \mathrm{~mL}$ of Auto-Induction Medium (ZYM-5052 ${ }^{48}$ ), incubated at $37^{\circ} \mathrm{C}$ with shaking (180 rpm) until OD $595 \sim 0.6$, and then incubated at $20^{\circ} \mathrm{C}$ with shaking $(180 \mathrm{rpm})$ overnight. The saturated culture was centrifuged $(4,000 \mathrm{rpm} / 10$ $\min / 4^{\circ} \mathrm{C}$ ), and resuspended in $40 \mathrm{~mL}$ of Lysis Buffer (50 mM Tris $\mathrm{pH}=8,500 \mathrm{mM} \mathrm{NaCl}, 20$ $\mathrm{mM}$ Imidazole, $10 \%$ Glycerol). Cells were lysed by passaging them six times using a microfluidizer. Lysates were centrifuged $\left(35,000 \mathrm{rpm} / 25 \mathrm{~min} / 4^{\circ} \mathrm{C}\right)$, and cleared lysates were added to washed Nickel-beads. After discarding the flow-through, beads were washed thrice with $5 \mathrm{~mL}$ of Lysis Buffer, and bound proteins were eluted with $1 \mathrm{~mL}$ of Elution Buffer in five fractions ( $50 \mathrm{mM}$ Tris $\mathrm{pH}=8,500 \mathrm{mM} \mathrm{NaCl}, 300 \mathrm{mM}$ Imidazole, 10\% Glycerol). Fractions were pooled together. $500 \mu \mathrm{g}$ of RT-Sen2 protein were used to isolate DNA (shown in ED Fig. 9B). DNA was extracted from the elution fraction twice with Phenol: Chloroform: Isoamyl-Alcohol (25:24:1, pH 8), and then nucleic acids were precipitated overnight at $4^{\circ} \mathrm{C}$ with isopropanol. Precipitated nucleic acids were centrifuged at $14,000 \mathrm{rpm} / 60 \mathrm{~min} / 4^{\circ} \mathrm{C}$. Pellets were resuspended once with $1 \mathrm{~mL}$ of $70 \%$ Ethanol, and centrifuged again at $14,000 \mathrm{rpm} / 60 \mathrm{~min} / 4^{\circ} \mathrm{C}$. Resultant pellets were air-dried (15 minutes), resuspended in $10 \mu \mathrm{L}$ of distilled water containing RNase $A(20 \mu \mathrm{g} / \mathrm{mL})$, and incubated at $37^{\circ} \mathrm{C}$ for 30 minutes. The sample was loaded on a $1 \mathrm{x}$-TBE:12\%-Polyacrylamide gel (with 1X-TBE buffer), electrophoresed at $70 \mathrm{~V}$ for 3.5 hours (constant voltage), and DNA was stained with ethidium bromide.

\section{SDS-PAGE and Immunoblot}

Samples from rrtT-3xFlag, rcaT-3xFlag, and control strains were suspended in 1x Laemmli buffer, and heated to $95^{\circ} \mathrm{C}$ for $10 \mathrm{~min}$. Proteins were separated by SDS-PAGE, and the gel was blotted to a PVDF membrane. Membranes were blocked for 1 hour (RT) with $5 \%$ skimmed milk in TBS-T (TBS-TM), and probed over-night at $4^{\circ} \mathrm{C}$ either in TBS-TM with a 1:1000 antiFlag antibody (Sigma-Aldrich; catalogue No F3165), or with a 1:10000 anti-LpoA antibody (loading control 28 ; wherever applicable, membranes were cut, with one half probed with anti- 
645 LpoA, and the other half with anti-Flag). Membranes were incubated for 1 hour with HRP-

646 conjugated secondary antibodies (1:5000, anti-mouse, Sigma-Aldrich Catalogue No A9044;

647 Flag, or 1:10000, anti-rabbit, Merck, Catalogue No GENA934; LpoA) in TBS-TM. After washing

648 with TBS-T, chemiluminescence substrate (GE-Healthcare) was added, and signal was

649 detected using X-ray films (Advantsta). X-ray films were then scanned at 300x300 dpi. Digital

650 images were cropped, and adjusted in Inkscape. Signal quantifications were done in ImageJ.

651

652 Retron-Eco9 identification

653 Retron-Sen2 components ( $r c a T$ and $r$ rtT gene products) were used as queries in pBLAST to

654 identify retron elements in the $E$. coli natural isolate collection ${ }^{24}$. Retron-Eco9 was selected as

655 having proteins homologous to both rcaT-Sen2 and rrtT. 


\section{EXTENDED DATA FIGURE LEGENDS}

\section{Extended Data Figure 1. Retron phenotypes and msDNA-Sen2 biosynthesis.}

658 (A) Perturbing msDNA-biogenesis leads to cold-sensitivity. STm wildtype and retron-deletion 659 strains were serially diluted and spotted on LB plates as in Fig. 1D. Plates were incubated at $66020^{\circ} \mathrm{C}, 25^{\circ} \mathrm{C}$, or $37^{\circ} \mathrm{C}$. Representative data shown from four independent experiments.

661 (B) Retron mutants grow slower in anaerobic conditions. Growth curves of STm wildtype and 662 retron-deletion strains were obtained by measuring OD578 in microtiter plates, under anaerobic conditions at $37^{\circ} \mathrm{C}$. Data plotted as in Fig. $2 \mathrm{C}$.

664 (C) Retron mutants are not affected in aerobic conditions. Experiment as in panel B, but strains 665 were grown aerobically.

666 (D) RNAse $\mathrm{H}$ and Exonuclease VII are involved in msDNA biosynthesis. msDNA was extracted 667 from STm wildtype and retron-deletion strains carrying plasmid p-retron- $\triangle S T M 14 \_4640$. 668 Extracted msDNA was electrophoresed in TBE-Polyacrylamide gels. A representative gel from 669 three independent experiments is shown.

\section{Extended Data Figure 2. Retron phenotype suppressing mutations inactivate rcaT.}

(A) Suppressor strains are reverted to wildtype growth in cold temperatures. Suppressors isolated from cold-sensitive STm mutants ( $\Delta r t T, \Delta x s e A, \Delta m s r m s d)$ were grown, serially diluted and spotted on LB plates as in Fig. 1D. Identified suppressor mutations are indicated.

(B) Progressive msd deletions constructed - in black the region deleted from the msrmsdRNA.

677 (C) Internal $m s d$ deletions from $\Delta 62$ to $\Delta 71$ suppress the cold-sensitivity of $\Delta x s e A$ cells, but induce cold-sensitivity in wildtype, presumably because they are antitoxin-deficient. Wildtype (WT) or $\Delta x s e A$ STm strains carrying msd deletions and the intact msrmsd region were grown, serially diluted and spotted on LB plates as in Fig. 1D. In contrast to all other internal deletions, the $\Delta 79: m s d$ mutant behaves as a full msrmsd deletion. Representative data shown from two independent experiments.

683 (D) Internal $m s d$ deletions (from $\Delta 62$ to $\Delta 71$ ) result in lower RcaT levels. rcaT-3xFlag tagged 684 STm strains carrying msd deletions and the WT STm strain were either grown in LB only at $68537^{\circ} \mathrm{C}$, or shifted to $20^{\circ} \mathrm{C}$ for 5 hours. Protein samples from strains were analysed by SDS686 PAGE and immunoblotting. LpoA levels ( $\alpha$-LpoA antibody) were used as a loading control. 687 Representative data shown from two independent experiments.

Extended Data Figure 3. RcaT is bacteriostatic at native levels, but becomes bactericidal 690 at higher levels

691 (A) RcaT inhibits growth at $15^{\circ} \mathrm{C}$ in STm. STm strains (WT and $\Delta m s r m s d$ ) were grown 692 overnight at $37^{\circ} \mathrm{C}$ and used to inoculate new cultures at $15^{\circ} \mathrm{C}$ for which the growth as 
693

694

695

696

697

698

699

700

701

702

703

704

705

706

707

708

709

710

711

712

713

714

715

716

717

718

719

720

721

722

723

724

725

726

727

728

729

monitored by measuring optical density (OD595). Data points represent the average of three measurements (biological replicates). Error bars denote standard deviation (if not shown, smaller than the symbols).

(B) RcaT is bacteriostatic at native levels in STm. STm strains (WT and $\Delta m s r m s d$ ) were grown at $15^{\circ} \mathrm{C}$, and viability curves were obtained by plating culture sample dilutions on LB plates to count colony forming units (CFU) per ml. Data points represent one experiment.

(C) Cold aggravates RcaT-mediated toxicity in E. coli. E. coli carrying plasmid p-rcaT or an empty vector were grown in ampicillin-LB for 5-6 hours at $37^{\circ} \mathrm{C}$, serially diluted and spotted on ampicillin-LB plates with or without arabinose (Ara). Representative data shown from three independent experiments.

(D) RcaT is bactericidal when overexpressed in E. coli. E. coli BW25113 strains carrying plasmid p-rcaT or an empty vector were grown in ampicillin-LB at $37^{\circ} \mathrm{C}$, and then cultures were transferred at $15^{\circ} \mathrm{C}$, and induced with arabinose. Viability curves were obtained by monitoring growth over time and plating culture samples on ampicillin-LB plates to count colony forming units (CFU) per ml. Data points represent the average of two experiments (biological replicates). Error bars denote standard deviation (if not shown, smaller than the symbol).

\section{Extended Data Figure 4. Retron functions as a toxin/antitoxin system in E. coli.}

(A) RcaT inhibition requires both msrmsd and rrtT in E. coli. E. coli with plasmids carrying retron-components were grown for $5-6$ hours at $37^{\circ} \mathrm{C}$ in spectinomycin-LB, serially diluted, spotted on spectinomycin-LB plates with or without arabinose, and plates were incubated at $37^{\circ} \mathrm{C}$. Only the intact retron could restore growth. RcaT expression is sufficient to inhibit growth. Representative data shown from three independent experiments.

(B) RcaT can be inhibited by msrmsd-rrtT also in trans. E. coli carrying binary combinations of plasmids p-rcaT, p-retron- $\Delta r c a T$, and empty vectors, were grown in LB with appropriate antibiotics, serially diluted and spotted as in A. Representative data shown from three independent experiments.

(C) RcaT inhibition requires RNAse $\mathrm{H}$ and Exo VII in E. coli. E. coli strains (WT, $\Delta x \operatorname{se} A, \Delta x s e B$, $\Delta r n h A)$ carrying plasmid p-retron- $\Delta r c a T$ or $p$-retron were grown, serially diluted and spotted as in A. Representative data shown from two independent experiments.

\section{Extended Data Figure 5. Antitoxin does not act on RcaT expression.}

(A) RcaT levels remain largely unaffected by antitoxin deletions. rcaT-3xFlag STm strains (WT and retron-deletions) and the STm untagged strain (native) were either grown in LB only at $37^{\circ} \mathrm{C}$, or shifted to $20^{\circ} \mathrm{C}$ for 5 hours. Protein samples from strains were analysed by SDSPAGE and immunoblotting. LpoA levels ( $\alpha$-LpoA antibody) were used as a loading control. Data shown from three independent experiments. 
(B) Quantification of RcaT-3xFlag signal from immunoblots in A using ImageJ (pixel-density).

731 Error bars depict standard deviation.

732 (C) Flag-Tagged RcaT retains its function. rcaT-3xFlag STm strains (wildtype-WT and retron733 deletions) and their untagged counterparts were grown for $5-6$ hours at $37^{\circ} \mathrm{C}$ in $\mathrm{LB}$, serially 734 diluted, spotted on LB plates, and incubated at $37^{\circ} \mathrm{C}, 25^{\circ} \mathrm{C}, 20^{\circ} \mathrm{C}$, or $15^{\circ} \mathrm{C}$. * denotes $\triangle S T M 14$ 4645::cat, which is used to co-transduce the scarless Flag-tagged rcaT (cotransduction verified by PCR). Representative data shown from two independent experiments.

\section{Extended Data Figure 6. RT and RcaT reciprocally pull down each other.}

739 Volcano plots of APs of RT-Sen2 (A) and RcaT (B) at $37^{\circ} \mathrm{C}$ and $20^{\circ} \mathrm{C}$ in wildtype and different 740 mutant backgrounds, performed as described in Fig. 3. The y-axis represents $p$-values of 741 identified peptides from two biological replicates in rrtT-3xFlag and rcaT-3xFlag IP samples, 742 and the $x$-axis represents the peptide-enrichment in the two samples compared to an untagged 743 STm strain control.

744

Extended Data Figure 7. Flag-tagged RrtT retains its function. rrtT-3xFlag STm strains (WT and retron-deletions) and their untagged counterparts were grown for $5-6$ hours at $37^{\circ} \mathrm{C}$ in LB, serially diluted, spotted on LB plates, and plates were incubated either at $37^{\circ} \mathrm{C}, 25^{\circ} \mathrm{C}$, $20^{\circ} \mathrm{C}$, or $15^{\circ} \mathrm{C}$.

\section{Extended Data Figure 8. Effects of tagging on retron-protein levels.}

751 Flag-tagging $r r t T$ does not alter retron protein expression (A), whereas flag-tagging rcaT decreases retron protein levels (B). Proteins in input (whole proteome) samples used for AP samples shown in Fig. 3 were quantified by mass spectrometry. Protein abundances in input samples of Flag-tagged strains were compared to input samples of untagged STm strains (xaxis). The $y$-axis represents the $p$-values of log2 fold-changes of quantified proteins. Data derived from two biological replicates.

\section{Extended Data Figure 9. RT interacts with msDNA.}

759 (A) Purification of protein RT-Sen2. An E. coli BL21 (DE3) CodonPlus-RIL strain carrying 760 plasmid p-msrmsd-rrtT-6xHis was used to purify protein RT-Sen2-6xHis (C-terminal fusion), 761 by nickel-column immobilized metal-affinity chromatography.

762 (B) His-tagging $r r t T$ does not affect its antitoxin activity. E. coli BL21-Al strains carrying binary 763 combinations of plasmids p-msrmsd-rrtT-6xHis, p-rcaT, and empty vectors, were grown for 57646 hours at $37^{\circ} \mathrm{C}$ in kanamycin-LB, serially diluted, spotted on kanamycin-LB plates with or 765 without arabinose, and plates were incubated at $20^{\circ} \mathrm{C}$. 
(C) Isolation of msDNA from purified RT-Sen2. Total DNA was extracted from $500 \mu \mathrm{g}$ of purified RT-Sen2-6xHis protein. Resultant DNA was electrophoresed on a TBEPolyacrylamide gel.

Extended Data Figure 10. E. coli Retron-Eco9 is similar to STm retron-Sen2.

(A) Retron-Eco9 has a similar operon structure to retron-Sen2. Retron-Eco9 contains msr, msd, rcaT and rrtT regions, which are $85 \%, 58 \%, 43 \%$, and $49 \%$ identical (first two nucleotide level, last two protein level) to the corresponding retron-Sen2 regions.

(B) msDNA-Eco9 is of similar structure to msDNA-Sen2. Models of msDNA-Sen2 and msDNA-

775 Eco9 are depicted, created using ${ }^{49}$.

(C) Retron-Eco9 is a TA system. E. coli BW25113 strains carrying plasmids p-rcaT-Eco9, pretron-Eco9, or an empty vector, were grown for $5-6$ hours at $37^{\circ} \mathrm{C}$ in chloramphenicol-LB, serially diluted, spotted on chloramphenicol-LB plates with or without arabinose, and plates were incubated at $37^{\circ} \mathrm{C}$. Representative data shown from three independent experiments.

(D) RcaT-Eco9 inhibition requires RNAse $\mathrm{H}$ and Exo VII in E. coli. E. coli BW25113 strains (wildtype, $\Delta x s e A, \Delta x s e B, \Delta r n h A$ ) carrying plasmid Ptac-msrmsd-Eco9, along with either PBADRT-Eco9, or PBAD-RT-RcaT-Eco9, were grown for 5-6 hours at $37^{\circ} \mathrm{C}$ in LB with appropriate antibiotics, serially diluted, spotted on LB plates with antibiotics, with/without arabinose, and plates were incubated overnight at $37^{\circ} \mathrm{C}$. Representative data shown from two independent experiments.

(E) msDNA-Eco9 biosynthesis requires RNase $\mathrm{H}$ and Exo VII. msDNA was extracted from $E$. coli BW25113 strains (wildtype, $\Delta x s e A, \Delta x s e B, \Delta r n h A$ ) carrying plasmid PBAD-msrmsd-RTEco9. Extracted msDNA were electrophoresed in a TBE-Polyacrylamide gel.

789 (F) The Ec RT cannot be activated as antitoxin by basal non-cognate msDNA (Se) levels (compare with Fig. 4B). E. coli BW25113 was co-transformed with plasmids carrying RT-RcaT combinations of retron-Sen2 (Se) and -Eco9 (Ec) (PBAD-RT-RcaT; Se-Se, Se-Ec, Ec-Se, or Ec-Ec - arabinose induction), and either plasmids carrying msrmsd (Ptac-msrmsd; Se, or Ec - IPTG induction) or an empty vector (-). Strains were grown for 5-6 hours at $37^{\circ} \mathrm{C}$ in LB with appropriate antibiotics, serially diluted, spotted on LB plates with antibiotics, with/without arabinose, and then incubated at $37^{\circ} \mathrm{C}$. Representative data shown from two independent experiments.

\section{SUPPLEMENTARY INFORMATION}

799 Supplementary Table 1. Proteomics data of RT-3xFlag/RcaT-3xFlag pull-downs.

800 Supplementary Table 2. Genotypes of bacterial strains used in this study.

801 Supplementary Table 3. Description of plasmids used in this study.

802 Supplementary Table 4. Description of construction of plasmids used in this study. 
803 Supplementary Table 5. List of primers used in this study.

\section{REFERENCES METHODS \& EXTENDED DATA FIGURE LEGENDS}

36. Datsenko, K. A. \& Wanner, B. L. One-step inactivation of chromosomal genes in Escherichia coli K-12 using PCR products. Proc. Natl. Acad. Sci. U. S. A. 97, 6640-6645 (2000).

37. Baba, T. et al. Construction of Escherichia coli K-12 in-frame, single-gene knockout mutants: The Keio collection. Mol. Syst. Biol. 2, 2006.0008 (2006).

38. Cherepanov, P. P. \& Wackernagel, W. Gene disruption in Escherichia coli: TcR and KmR cassettes with the option of Flp-catalyzed excision of the antibiotic-resistance determinant. Gene 158, 9-14 (1995).

39. Liu, H. \& Naismith, J. H. An efficient one-step site-directed deletion, insertion, single and multiple-site plasmid mutagenesis protocol. BMC Biotechnol. 8, 91 (2008).

40. Wang, H. et al. Improved seamless mutagenesis by recombineering using ccdB for counterselection. Nucleic Acids Res. 42, e37-e37 (2014).

41. Chung, C. T., Niemela, S. L. \& Miller, R. H. One-step preparation of competent Escherichia coli: transformation and storage of bacterial cells in the same solution. Proc. Natl. Acad. Sci. 86, 2172-2175 (1989).

42. Chassy, B. Transformation of bacteria by electroporation. Trends Biotechnol. 6, 303-309 (1988).

43. Green, M. R. \& Sambrook, J. Preparation of Plasmid DNA by Alkaline Lysis with Sodium Dodecyl Sulfate: Minipreps. Cold Spring Harb. Protoc. 2016, pdb.prot093344 (2016).

44. Hughes, C. S. et al. Single-pot, solid-phase-enhanced sample preparation for proteomics experiments. Nat. Protoc. 14, 68-85 (2019).

45. Mateus, A. et al. Thermal proteome profiling in bacteria: probing protein state in vivo. Mol. Syst. Biol. 14, e8242 (2018).

46. Ritchie, M. E. et al. limma powers differential expression analyses for RNA-sequencing and microarray studies. Nucleic Acids Res. 43, e47-e47 (2015).

47. Deatherage, D. E. \& Barrick, J. E. Identification of Mutations in Laboratory-Evolved Microbes from Next-

48. Studier, F. W. Protein production by auto-induction in high-density shaking cultures. Protein Expr. Purif. Generation Sequencing Data Using breseq. in Engineering and Analyzing Multicellular Systems: Methods and Protocols (eds. Sun, L. \& Shou, W.) 165-188 (Springer New York, 2014). doi:10.1007/978-1-49390554-6_12 41, 207-234 (2005).

49. Zuker, M. Mfold web server for nucleic acid folding and hybridization prediction. Nucleic Acids Res. 31, 3406-3415 (2003).

50. Uehara, T., Parzych, K. R., Dinh, T. \& Bernhardt, T. G. Daughter cell separation is controlled by cytokinetic ring-activated cell wall hydrolysis. EMBO J. 29, 1412-1422 (2010).

51. Guzman, L. M., Belin, D., Carson, M. J. \& Beckwith, J. Tight regulation, modulation, and high-levels expression by vectors containing the arabinose PBAD promoter. J. Bacteriol. 177, 4121-4130 (1995). genetic studies. DNA Res. 12, 63-68 (2005). interactions during infection. Preprint at https://doi.org/10.1101/2020.05.04.075937 (2020). 
bioRxiv preprint doi: https://doi org/10.1101/2020.06.22 160168; this version posted June 22, 2020. The copyright holder for this pre (which was not certified by peer review) is the author/funder, yho has granted bioRxiv a license to display the preprint in perpetuity made arailgylelver aCC-BY 4.0 International license.

A

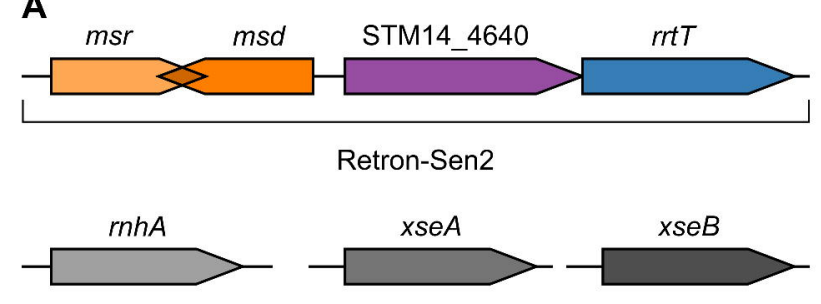

B
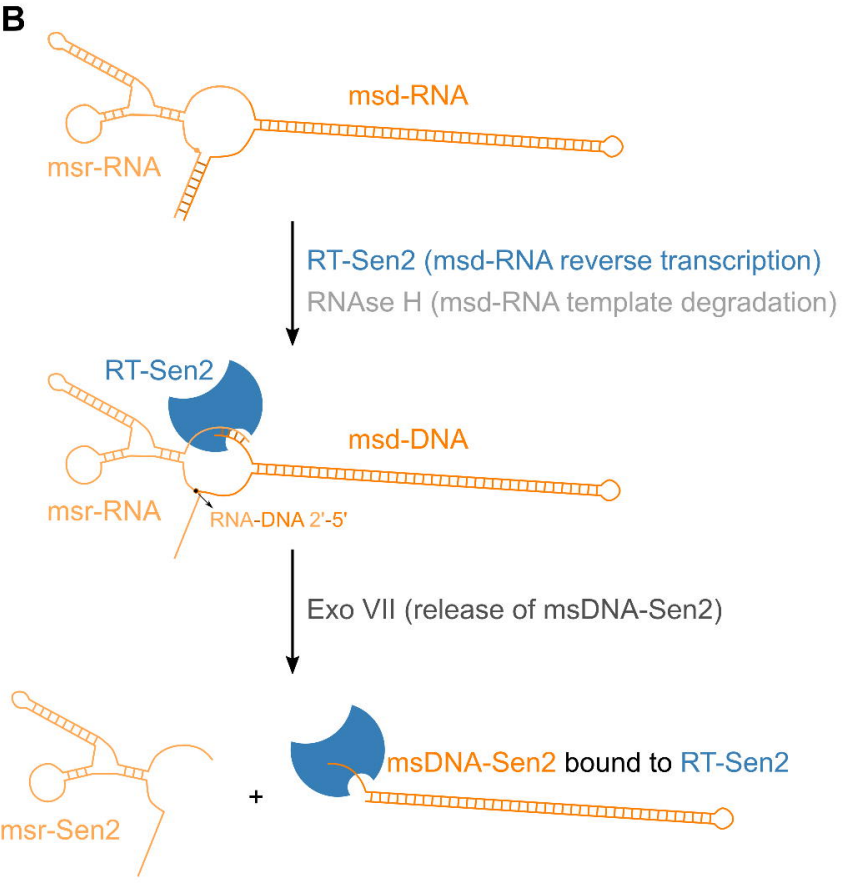

C

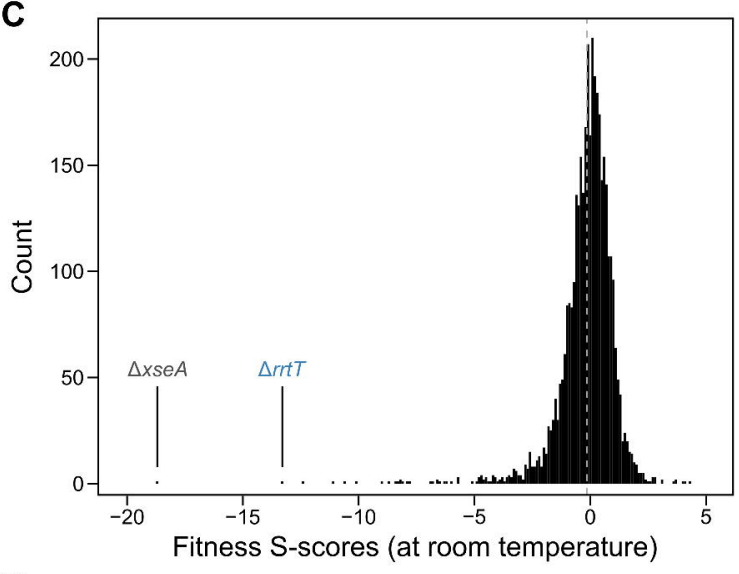

D

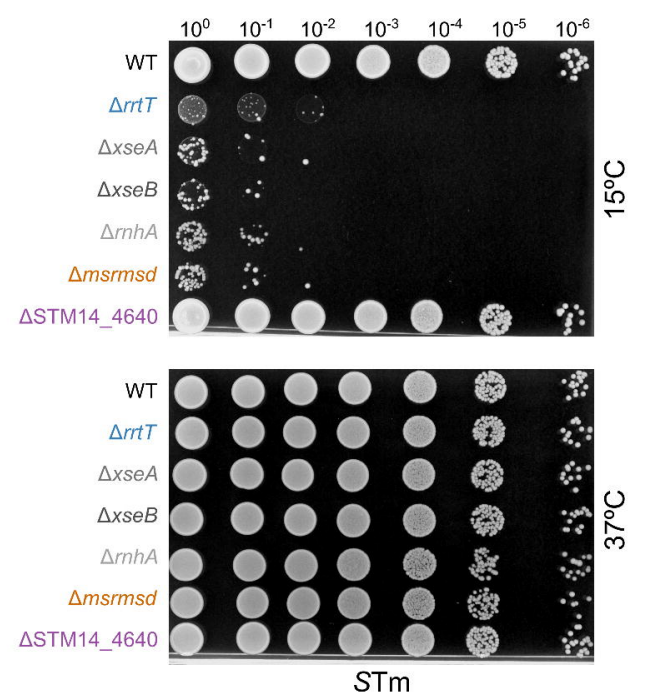


bioRxiv preprint doi: $\mathrm{https} / /$ doi org/10.1101/2020.06 22.160168; this version posted June 22, 2020. The copyright holder for this pre (which was not certified by peer review) is the author/funder, who has granted bioRxiv a license to display the preprint in perpetuity made arailegle reedei2aCC-BY 4.0 International license.

A

Suppressor selection at $15^{\circ} \mathrm{C}$

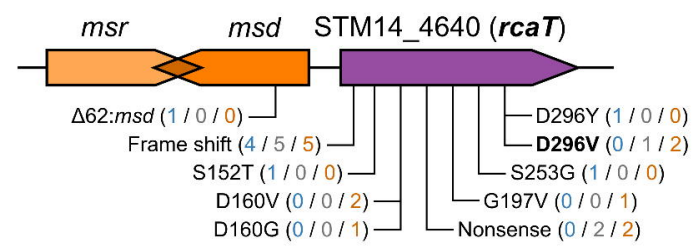

B

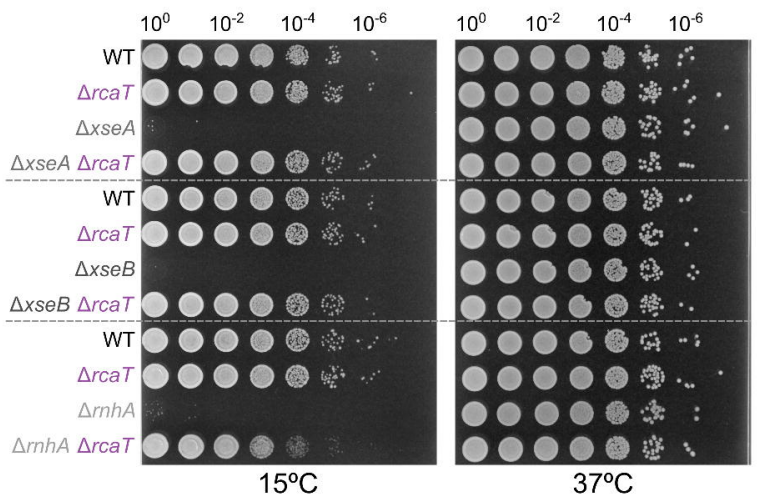

C

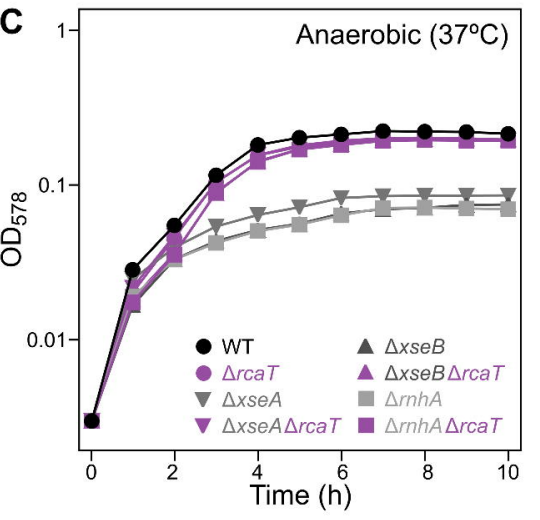

D E. coli BL21-AI

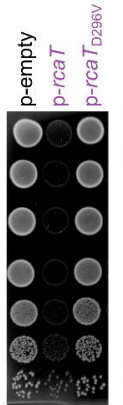

+Ara

mutation $(\mathrm{n} / \mathrm{n} / \mathrm{n})$

$$
\Delta r r t T
$$

$\triangle x s e A$

$\Delta m s r m s d$ 
bioRxiv preprint doi: https://doi.org/10.1101/2020.06.22.160168; this version posted June 22, 2020. The copyright holder for this pre (which was not certified by peer review) is the author/funder, who has granted bioRxiv a license to display the preprint in perpetuity made avatipble under कु 3

\section{A}

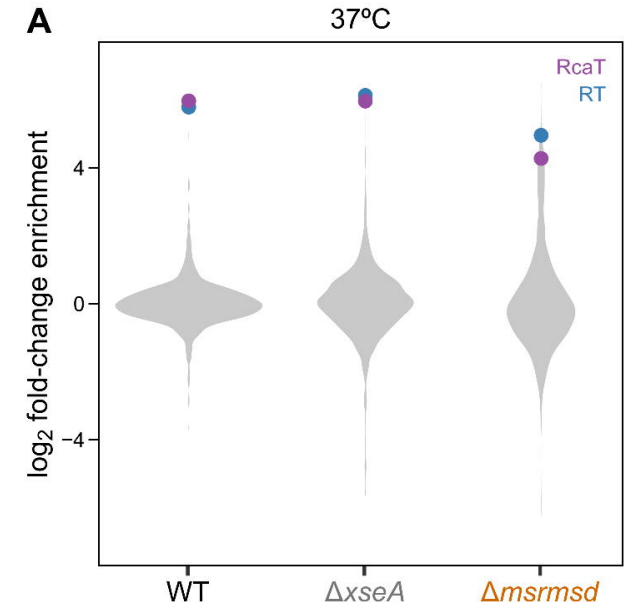

c

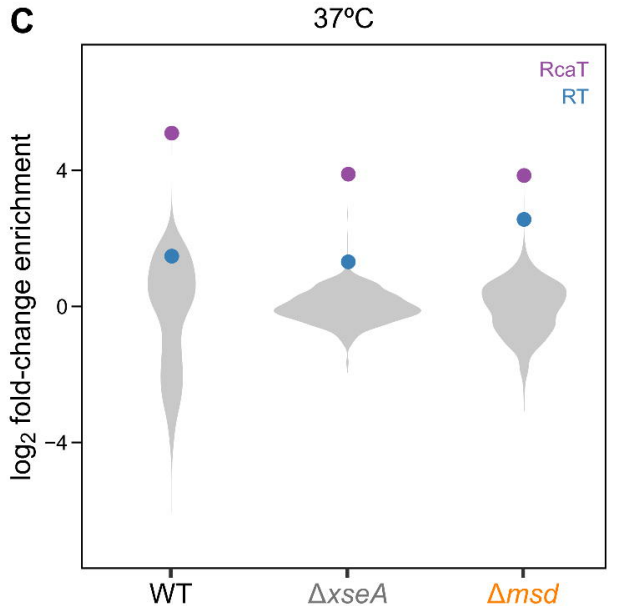

B

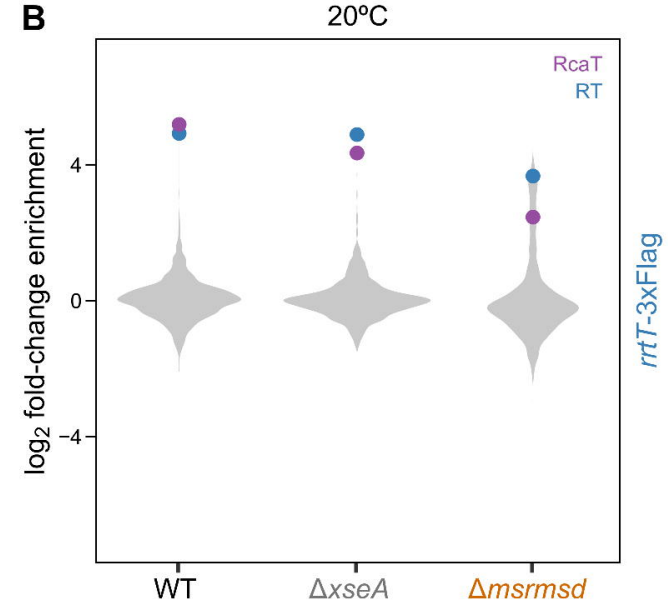

。

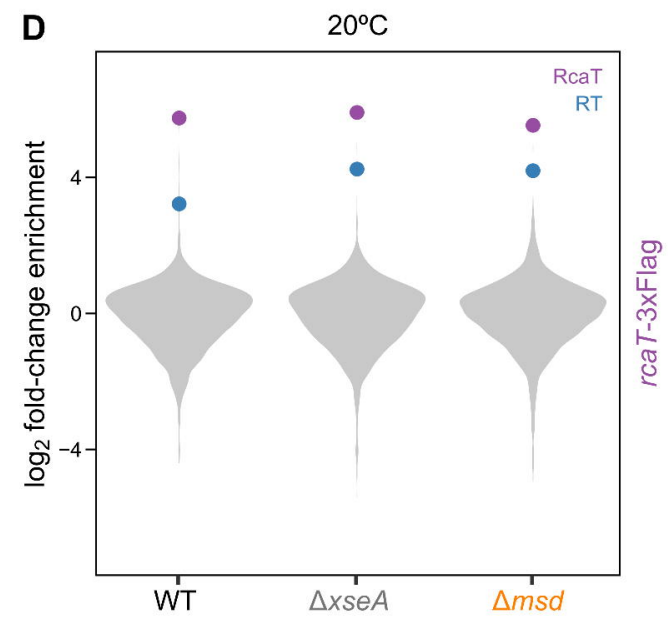


bioRxiv preprint doi: https://doi org/10.1101/2020.06.22 160168; this version posted June 22, 2020. The copyright holder for this pre (which was not certified by peer review) is the author/funder, who has granted bioRxiv a license to display the preprint in perpetuity made avilable under ${ }^{\mathrm{C}}$

A

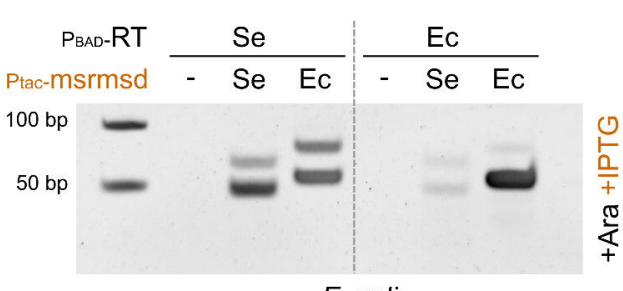

E. coli

B

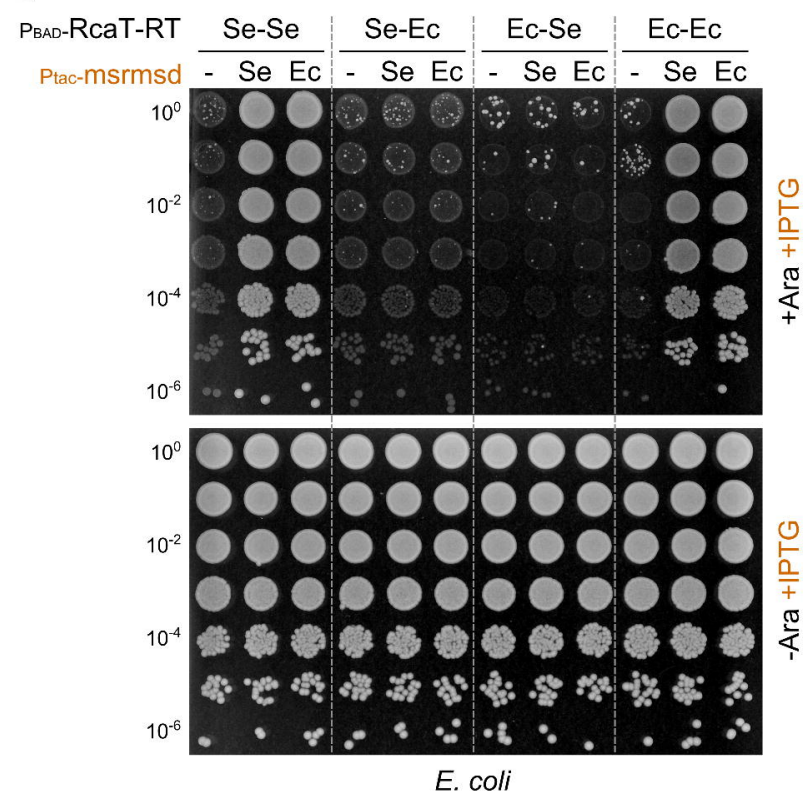




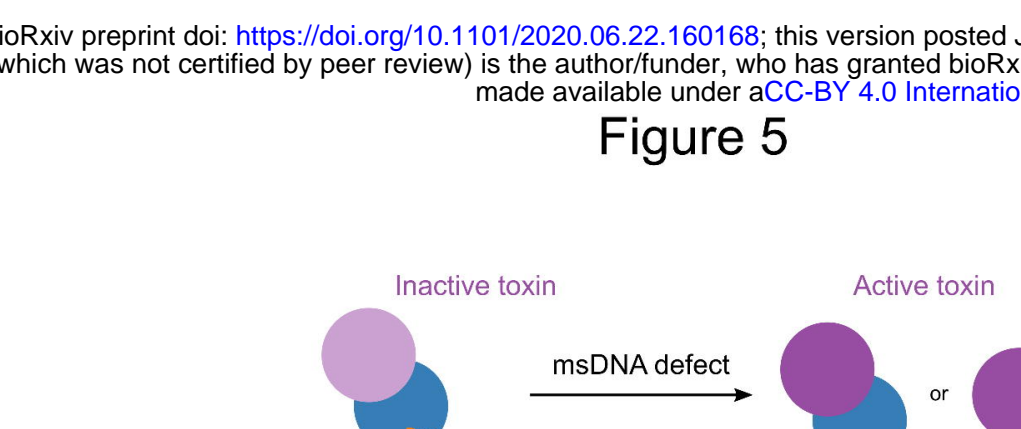


bioRxiv preprint doi: https://doi.org/10.1101/2020.06.22 160168. this version posted June 22, 2020. The copyright holder for this pre (which was not certified by peer review) is the author/funder, who has granted bioRxiv a license to display the preprint in perpetuity made available under aCC-BY 4.0 International license.

ED Figure 1

A
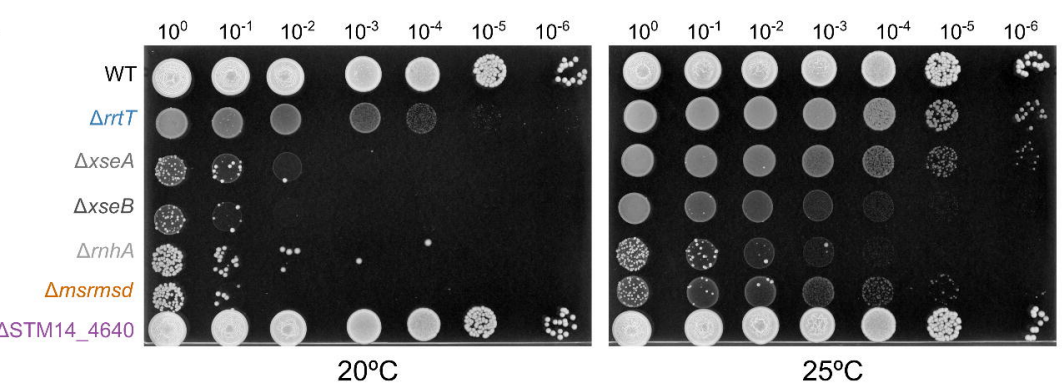

$25^{\circ} \mathrm{C}$
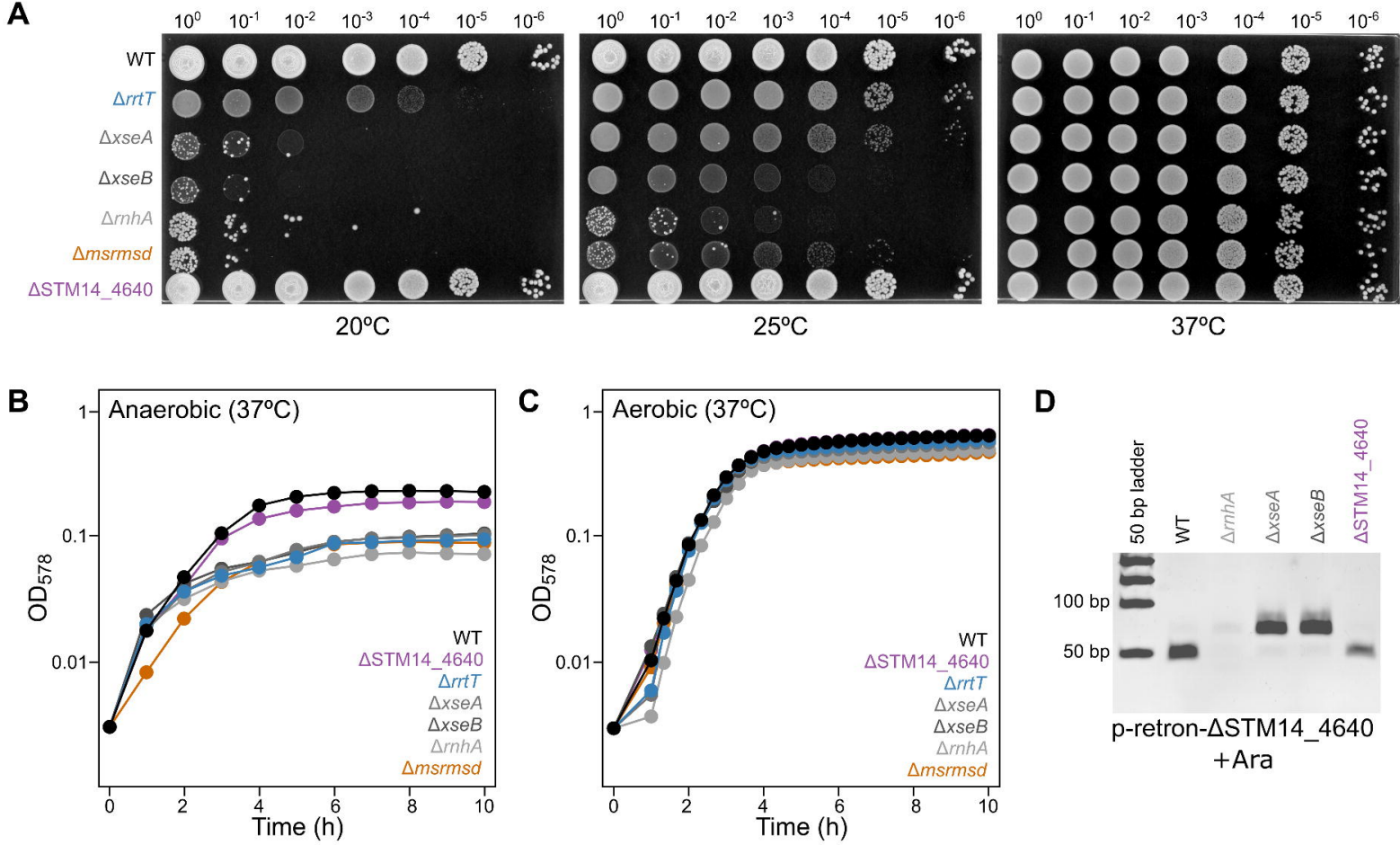
bioRxiv preprint doi: https://doi.org/10.1101/2020.06.22.160168; this version posted June 22, 2020. The copyright holder for this pre

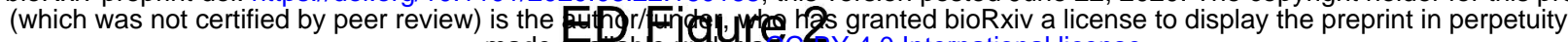
made avditable udet aec-BY 4.0 International license.

A $37^{\circ} \mathrm{C}$

$15^{\circ} \mathrm{C}$ WT 0000 \% 00002

\begin{tabular}{|c|c|}
\hline $0000095^{\circ}$ & \\
\hline J000खs & 0000 \\
\hline 1000098 & 50000 \\
\hline 1000004 & 00000 \\
\hline $00000 \%$ & 000008 \\
\hline $0000090:-2$ & 00000 \\
\hline $000006 \%$ & 0000 \% \\
\hline 0000098 & 000009 \\
\hline $000003 \div$ & $00000:$ \\
\hline
\end{tabular}

Mutation

WT

rcaT (frame shift)

rcat (frame shift)

rcaT (S152T)

rcat (S253G)

$\triangle 62: m s d$

$\operatorname{rcaT}(\mathrm{D} 296 \mathrm{Y})$

rcaT (frame shift)

rcaT (frame shift)

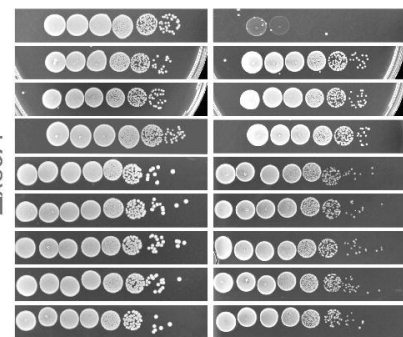

WT
rcaT (frame shift)

rcat (frame shift)

rcat (D296V)

rcat (frame shift)

rcat (nonsense; E180*)

rcat (frame shift)

rcaT (frame shift)

rcat (nonsense; E180*)

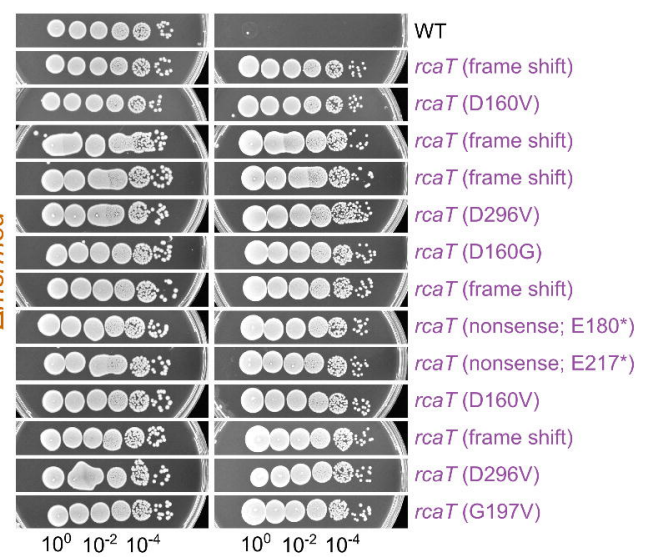

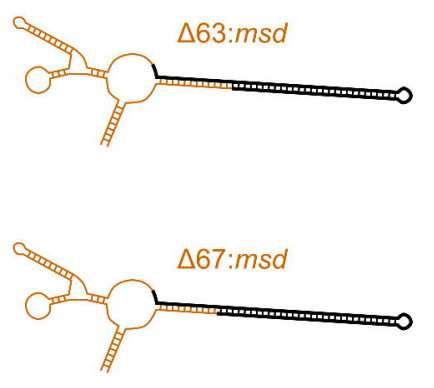
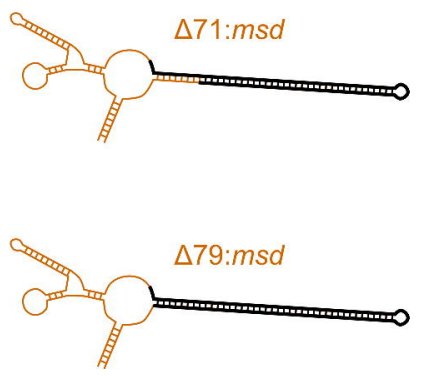

C

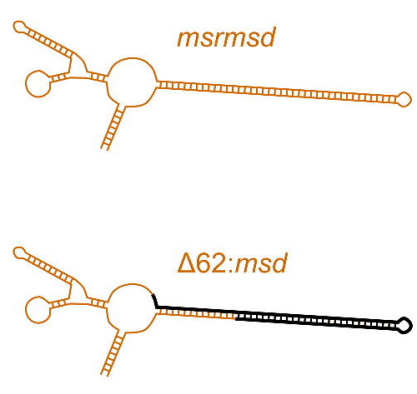

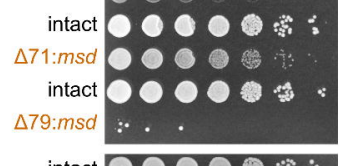

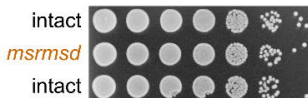

intact 00003

intact 00000 찬

$\triangle 63: m s d 00000$

intact 00000

$\triangle 67: m s d 00000$ :

intact 00000 :

$\Delta 71: m s d 00000$ 연

intact 00000 is

$\Delta 79: m s d 0000$ 녕 :

WT

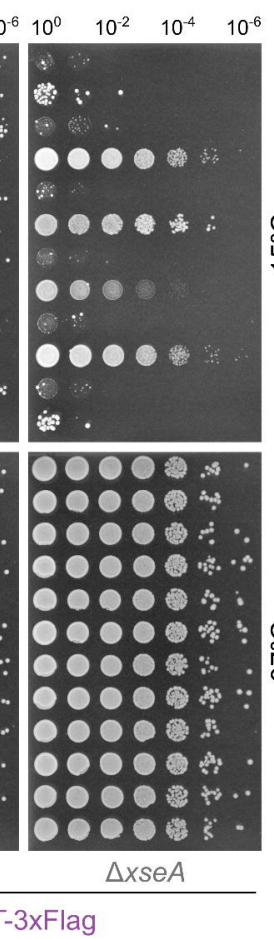

D

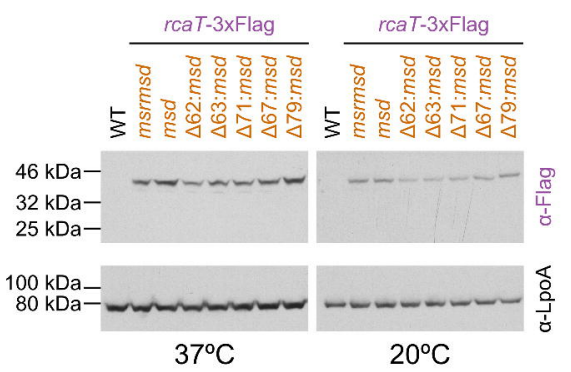


bioRxiv preprint doi: https://doi org/10.1101/2020.06.22.160168; this version posted June 22, 2020. The copyright holder for this pre (which was not certified by peer review) is the author/funder, who has granted bioRxiv a license to display the preprint in perpetuity made avajable.under aC ${ }^{\mathrm{B}}$

A

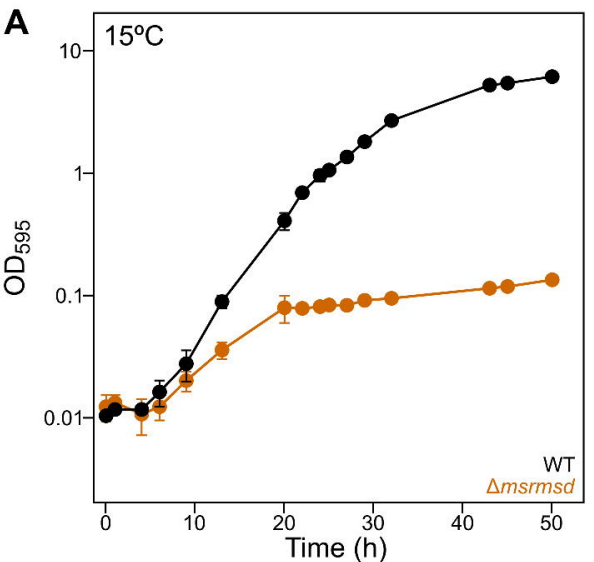

C

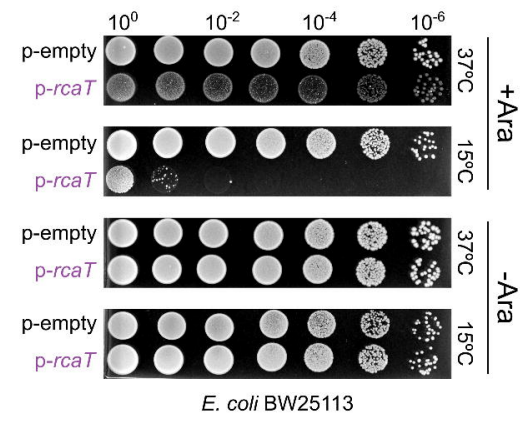

B

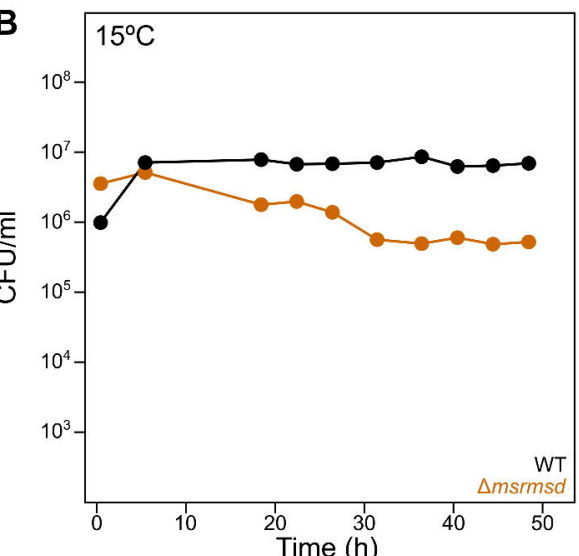

D

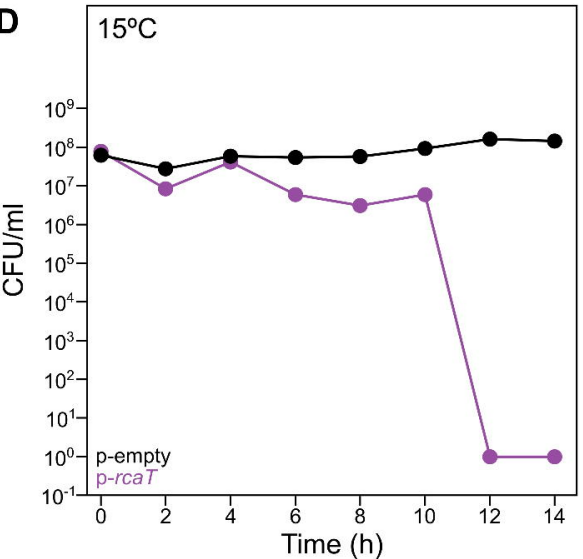


ercion nocted June 22,020 The convriaht holder for thic nre (which was not certified by peer review) is the author/funder, who has granted bioRxiv a license to display the preprint in perpetuity made evitable under acc $\mathrm{B}$

A
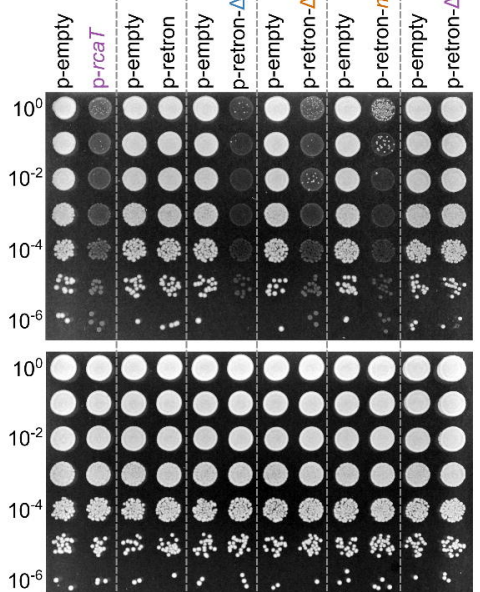

Retron-TA function in cis
B

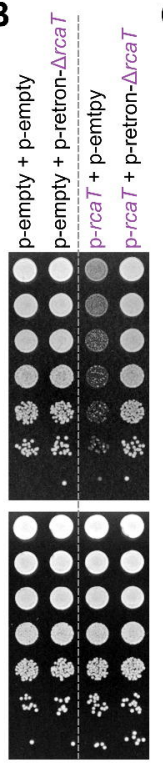

In trans
C

$\stackrel{\pi}{\frac{\pi}{4}}$

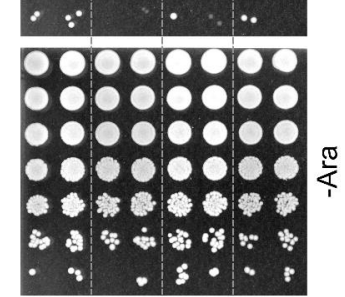

ExoVII/Rnase $\mathrm{H}$

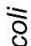

แ่ 
bioRxiv preprint doi: $\mathrm{https} \cdot / /$ doi org/10.1101/2020.06.22 160168. this version posted June 22,2020 . The copyright holder for this pre (which was not certified by peer review) is the author/funder, who has granted bioRxiv a license to display the preprint in perpetuity made

A

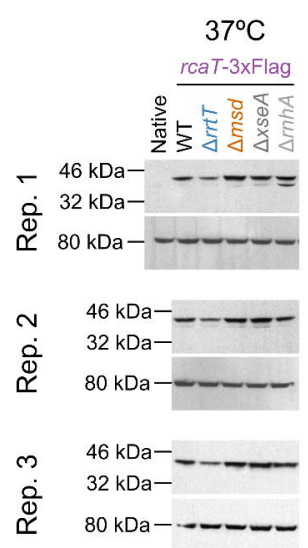

$46 \mathrm{kDa}--0--\alpha$-Flag

$32 \mathrm{kDa}-$

$80 \mathrm{kDa}$
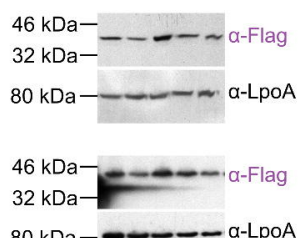

$80 \mathrm{kDa}-\boldsymbol{0}-\boldsymbol{-} \alpha-\mathrm{LpoA}$

\section{B}

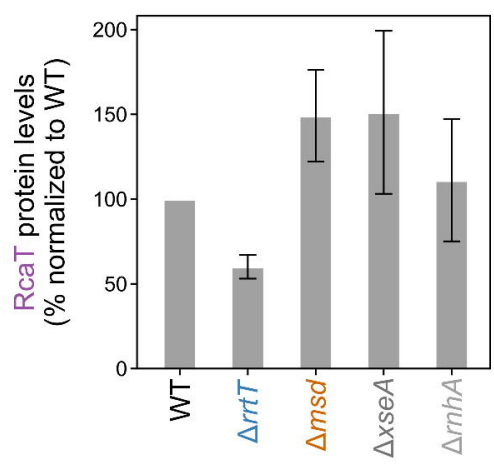

C

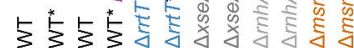
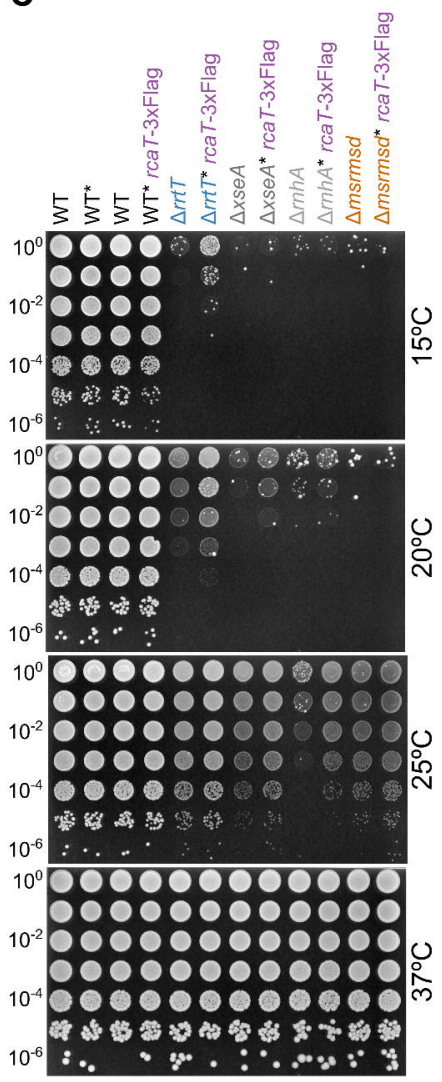
bioRxiv preprint doi: https://doi.org/10.1101/2020.06.22.160168; this version posted June 22, 2020. The copyright holder for this pre (which was not certified by peer review) is the authorffunder, who has granted bioRxiv a license to display the preprint in perpetuity made atablerail gutuce-6o 4.0 International license.

A $r$ rtT-3xFlag

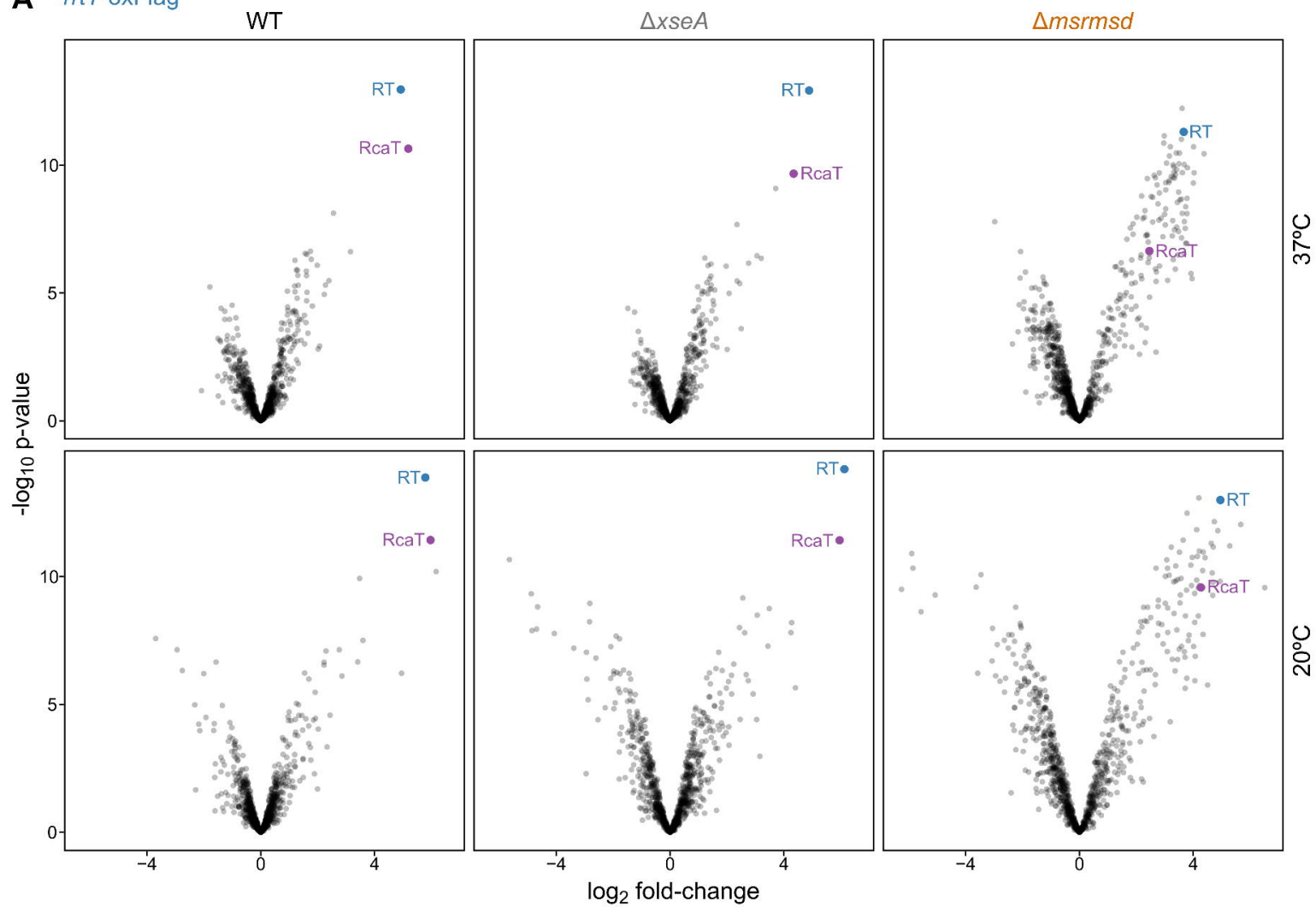

B rcat-3xFlag

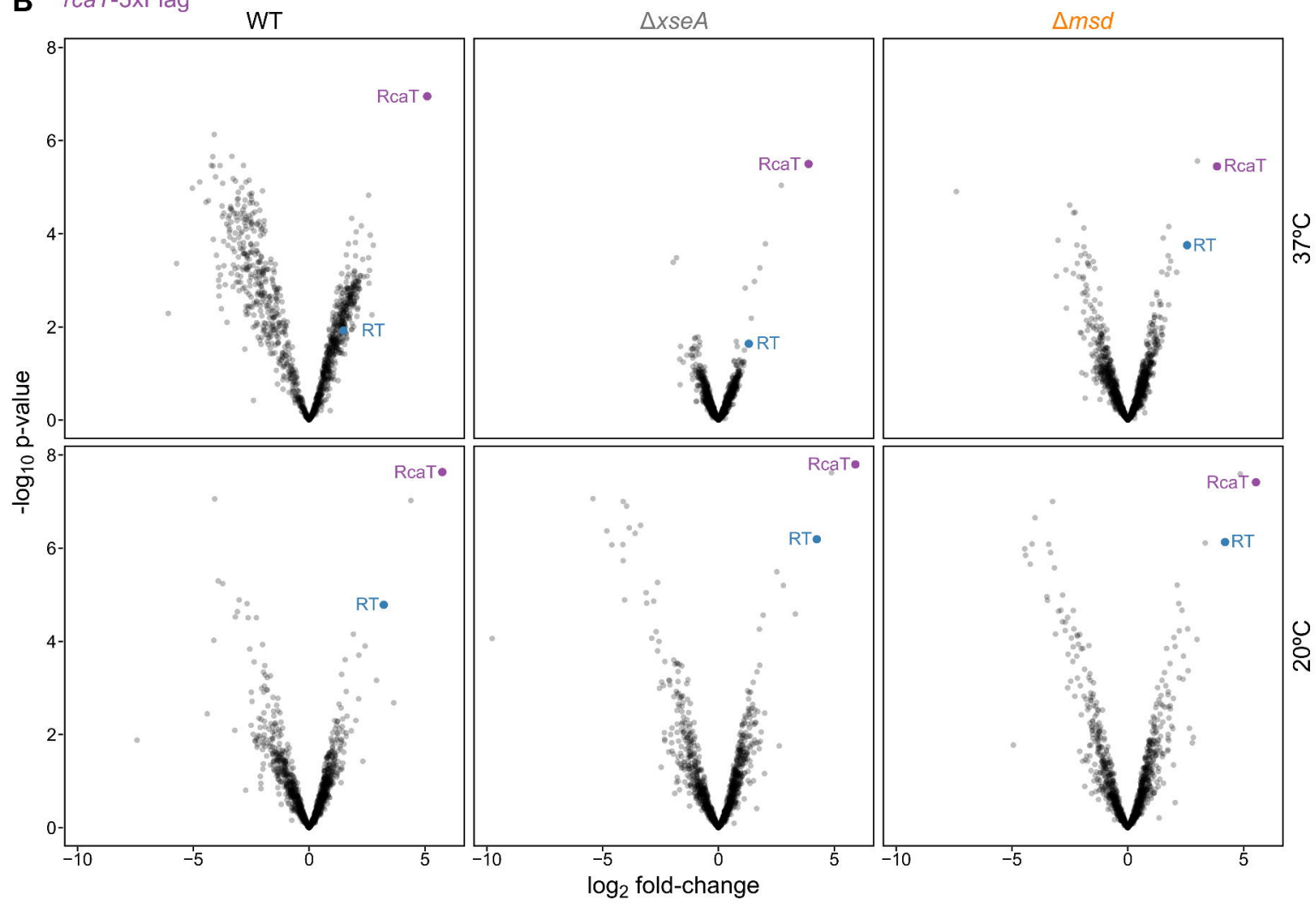


bioRxiv preprint doi: https//doi.org/10.1101/2020.06.22.160168; this version posted June 22, 2020. The copyright holder for this pre (which was not certified by peer review) is the author/funder, who has granted bioRxiv a license to display the preprint in perpetuity

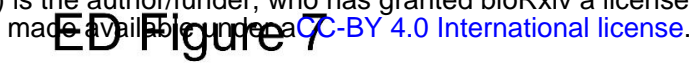

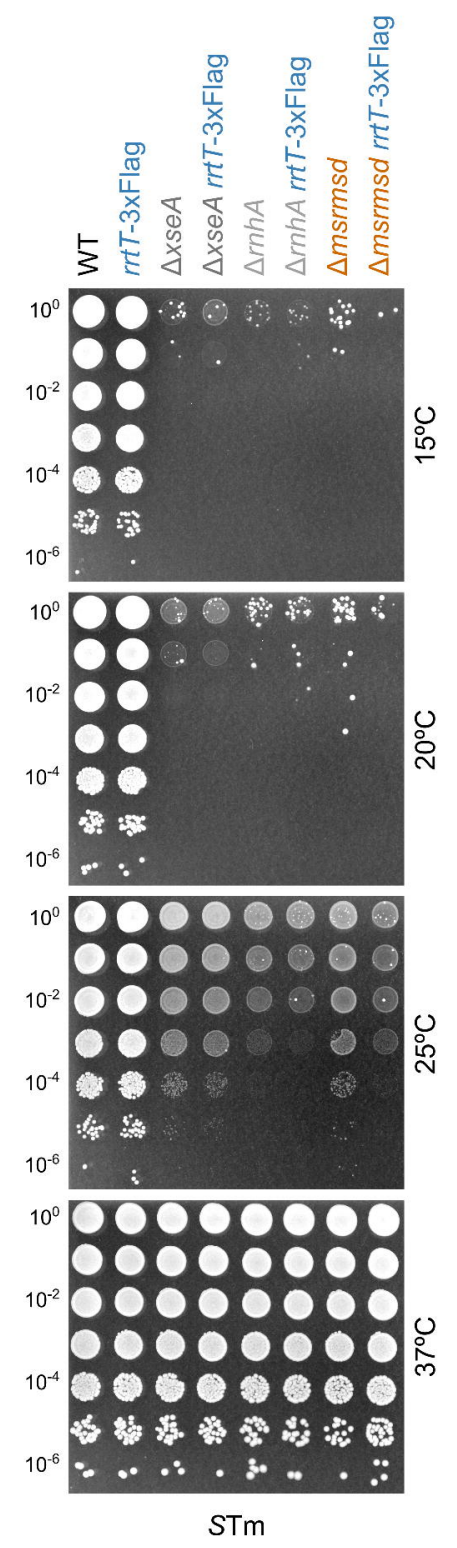


bioRxiv preprint doi: https://doi.org/10.1101/2020.06.22.160168; this version posted June 22, 2020. The copyright holder for this pre (which was not certified by peer review) is the auther/funder, who has granted bioRxiv a license to display the preprint in perpetuity made t迥olfiged kec 8 Y 4.0 International license.

A rrtT-3xFlag

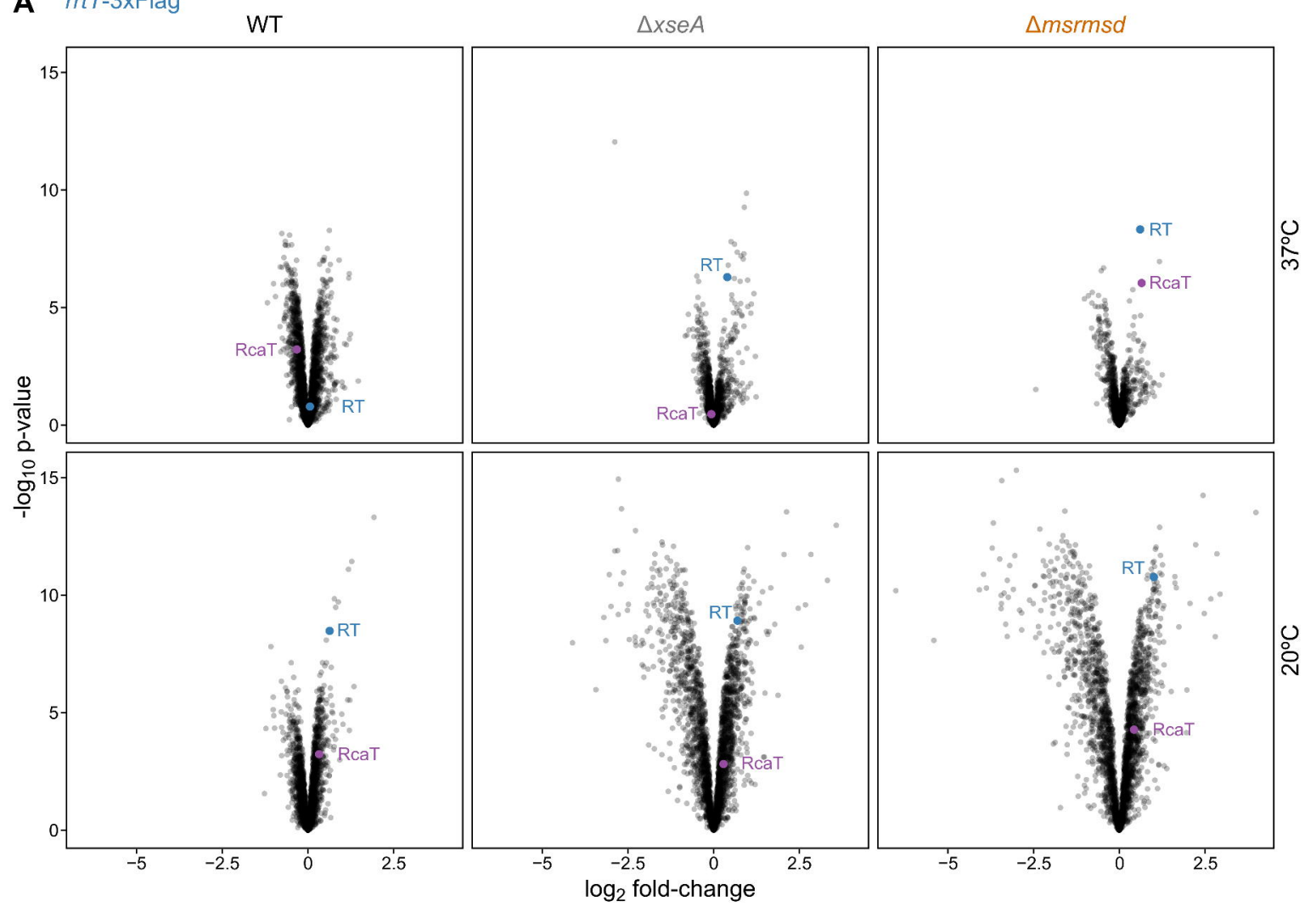

B rcat-3xFlag

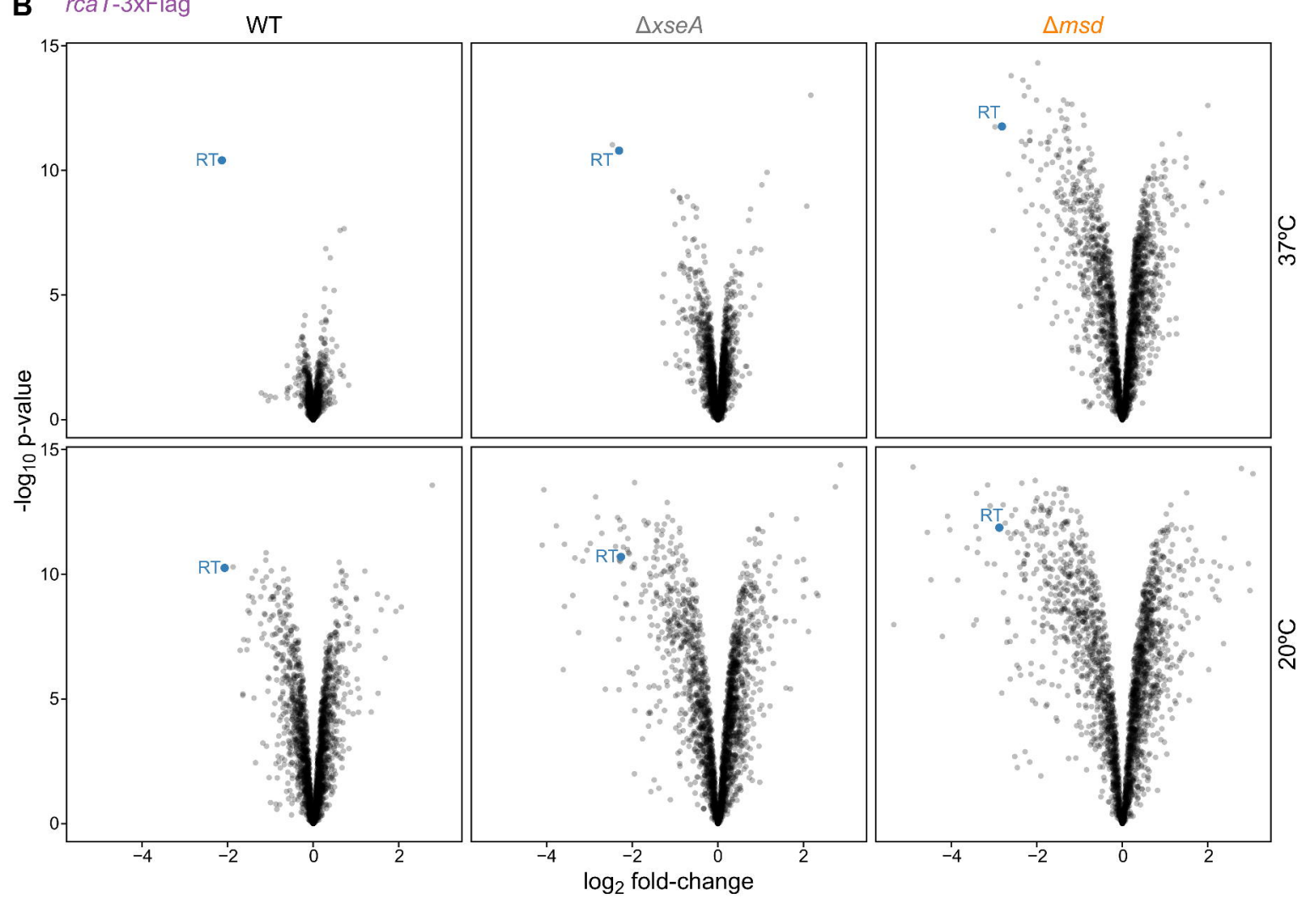


bioRxiv preprint doi: https://doi.org/10.1101/2020.06.22.160168; this version posted June 22, 2020. The copyright holder for this pre (which was not certified by peer review) is theaptho founder whodas granted bioRxiv a license to display the preprint in perpetuity

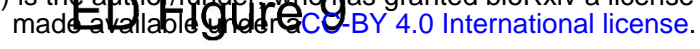

A

$32 \mathrm{kDa}$

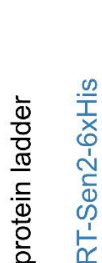

B

B

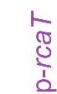

$10^{-2} \mathrm{C}$

을

है

O

$10^{-4}$

0

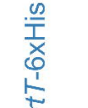

C

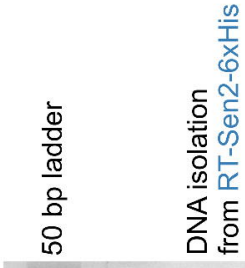

$10^{-6}$

$10^{2}$

$10^{-2} \bigcirc$

$10^{-4}$ 중

$10^{-6}$

E. coli $\mathrm{BL} 21-\mathrm{Al}\left(20^{\circ} \mathrm{C}\right)$

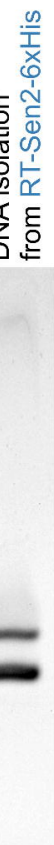


bioRxiv preprint doi: https://doi.org/10.1101/2020.06.22.160168; this version posted June 22, 2020. The copyright holder for this pre

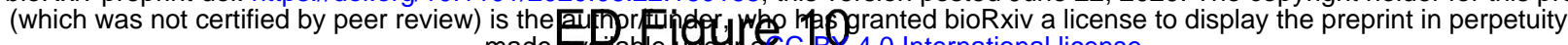
made avallable Coder céc- 4.0 International license.

A
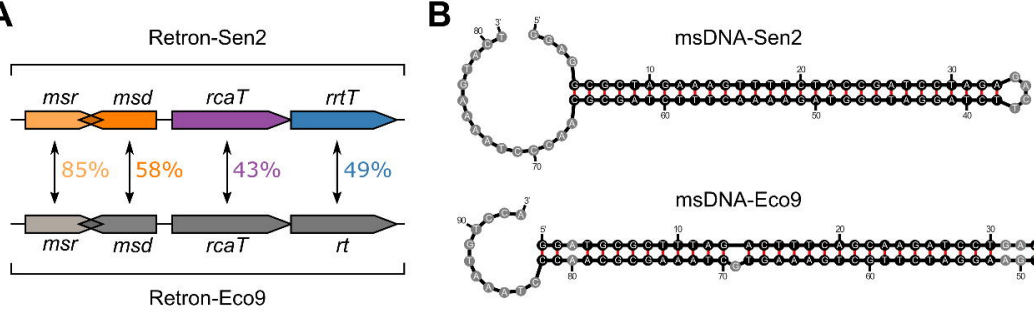

msDNA-Eco9

2

D Ptac-msrmsd-Eco9

PaAd-RT-Eco9

PвAD-RCaT-RT-Eco9

WT $\overline{\Delta x \operatorname{seA}} \overline{\Delta x \operatorname{sse}} \overline{\Delta r n h A}$

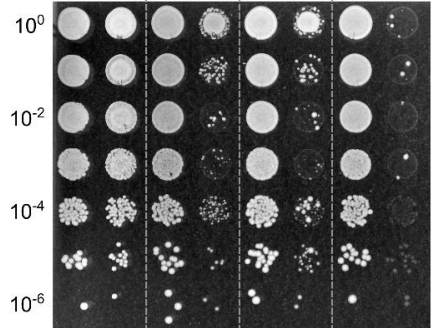

$\stackrel{\frac{\pi}{4}}{+}$

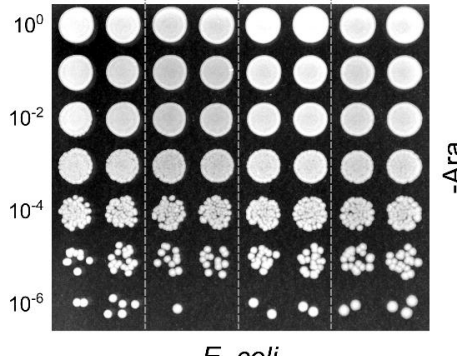

E. coli

E

PBad-msrmsd-RT-Ecog
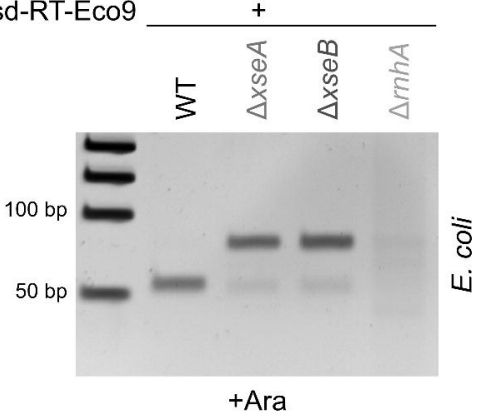

10
C

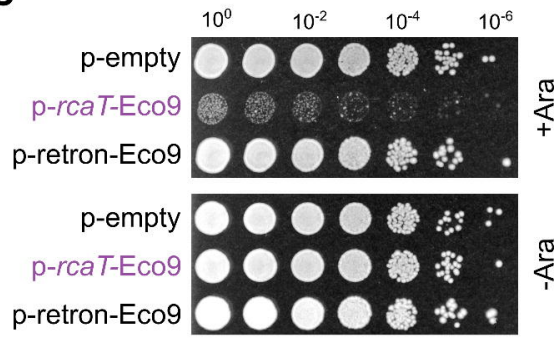

E. coli

\begin{tabular}{rc|c|c|c} 
Paad-RcaT-RT & Se-Se & Se-Ec & Ec-Se & Ec-Ec \\
\cline { 2 - 3 } & - Stac-msrmsd Ec & - Se Ec & - Se Ec & - Se Ec
\end{tabular}

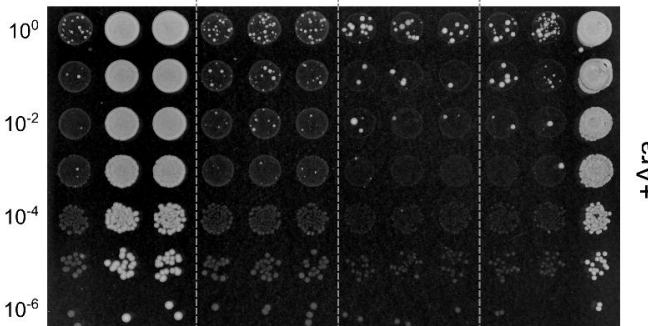

000000000000 00000000000 100000000000 1000 $\stackrel{\frac{\pi}{4}}{i}$

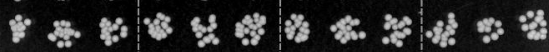

E. coli 